Portland State University

PDXScholar

$1-1-2010$

\title{
Hierarchical Image Analysis and Characterization of Scaling Effects in Remote Sensing
}

Craig David Ducey

Portland State University

Follow this and additional works at: https://pdxscholar.library.pdx.edu/open_access_etds

Let us know how access to this document benefits you.

\section{Recommended Citation}

Ducey, Craig David, "Hierarchical Image Analysis and Characterization of Scaling Effects in Remote Sensing" (2010). Dissertations and Theses. Paper 399.

https://doi.org/10.15760/etd.399

This Thesis is brought to you for free and open access. It has been accepted for inclusion in Dissertations and Theses by an authorized administrator of PDXScholar. Please contact us if we can make this document more accessible: pdxscholar@pdx.edu. 
Hierarchical Image Analysis and

Characterization of Scaling Effects in Remote Sensing

by
Craig David Ducey

Craig David Ducey

A thesis submitted in partial fulfillment of the requirements for the degree of

\author{
Master of Science \\ in \\ Geography \\ Thesis Committee: \\ Keith Hadley, Chair \\ Jiunn-Der Duh \\ Andrew Fountain
}

Portland State University

(C)2010 


\begin{abstract}
The effects of scale influence all aspects of spatial analysis and should be expressly considered early in research planning. Remotely sensed images provide unique landscape perspectives and possess several features amenable to dealing with scale. In particular, images can be segmented into image objects representative of landscape features and structured as nested hierarchies for evaluating landscape patterns across a range of scales. The objectives of this research are to evaluate methods for: 1) characterizing candidate image objects to inform the selection of user-supplied segmentation parameters and 2) exploring the multi-scale structure of landscape patterns for defining and describing potentially important scales for conducting subsequent geospatial and ecological investigations.

I followed a recursive strategy to develop an image hierarchy using a corrected version of the normalized difference vegetation index (NDVIc) derived from a Landsat ETM+ satellite image over a complex, forested landscape at Lava Cast Forest (LCF), Oregon. At each scale level, I calculated an objective function based on within-object variance and spatial autocorrelation to distinguish between alternative image objects created with the region-merging segmentation algorithm available in the Definiens Developer 7 software. Segmentation quality was considered highest for results exhibiting the lowest overall within-object variance and between-object spatial autocorrelation. I then applied geographical variance
\end{abstract}


analysis to calculate the independent contribution and relative variability of each level in the hierarchy to evaluate the scene's spatial structure across scales.

My results reveal overall trends in image object spatial variance consistent with scaling theory, but suggest judging image object quality without sampling the entire range of segmentation parameters is insufficient. Statistical limitations of the spatial autocorrelation coefficient at small sample sizes constrained the number of possible hierarchy levels within the image spatial extent, preventing identification of larger-scale landscape patterns.

Geographical variance analysis results show patterns in vegetation conditions at LCF possess a multi-scaled structure. Three levels exhibiting high variance relative to the entire hierarchy coincide with abrupt transitions in the slopes of within-object variance and spatial autocorrelation trends, which I interpreted as scale thresholds potentially important for relating landscape patterns and processes. These methods provide an objective, object-oriented approach for addressing scale issues within heterogeneous landscapes using remote sensing. 


\section{DEDICATION}

For my wife, Maggie Hodny, who provided unwavering support and encouragement throughout this entire process. 


\section{ACKNOWLEDGEMENTS}

I owe my highest gratitude to my academic advisor, Keith Hadley, for reviewing this document and providing a deeper appreciation for the fundamental concepts common to landscape ecology and remote sensing.

I acknowledge the members of my graduate advising committee, Geoffrey Duh and Andrew Fountain, for their commitment to my academic success.

This research would not be possible without the support and advocacy of my colleagues at the Bureau of Land Management and US Forest Service. In particular, I thank Terry Hobbs, Arthur Miller, Duane Dippon, Ryan Kelley, Shelley Moore, Kiet Nguyen, Dan Wickwire, and my mentors Jeff Nighbert, Jim Alegria, and Melinda Moeur.

Thank-you to Cory Owens, Josh Owens, Mick Thomure, and Jennifer Karps for their valuable insight and assistance. 
TABLE OF CONTENTS

ABSTRACT

Ecosystems as Spatial Hierarchies ............................................................................ 2

Multi-Scale Evaluations of Landscape Patterns with Remotely Sensing ...................... 6

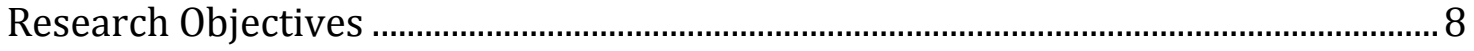

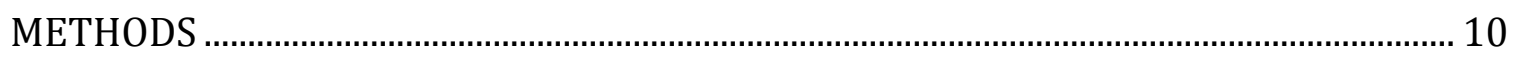

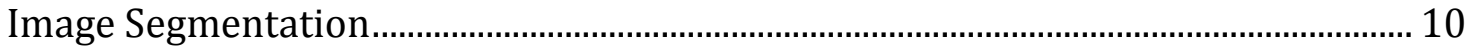

An Objective Function for Evaluating Segmentation Quality …………………........... 14

Geographic Variance Analysis .................................................................................... 17

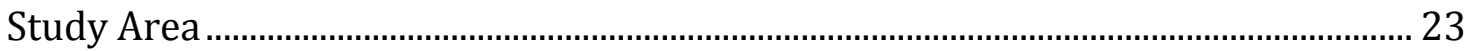

Image Processing ..................................................................................................... 26

Image Segmentation and Hierarchical Image Analysis ................................................ 28

RESULTS.................................................................................................................. 30

Pixel-Level Patterns in NDVIc.................................................................................. 30

The Effects of Alternative Parameters on Segmentation Results................................. 33

Distribution of Variance Across Scales .......................................................................... 41

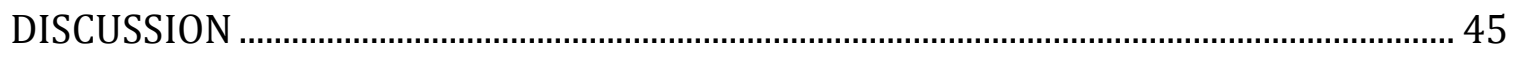

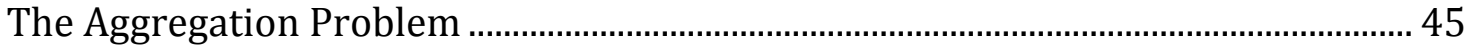

The Scaling Problem.................................................................................................. 49

Methodological Considerations ................................................................................. 54

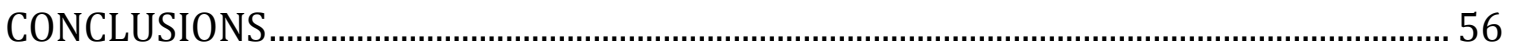

LITERATURE CITED ………………………………………………………….... 59

APPENDIX A: Example Geographical Variance Analysis ................................................. 66 


\section{LIST OF TABLES}

Table 1. Summary statistics for image segmentations possessing the lowest combined normalized Moran's I spatial autocorrelation coefficients and mean weighted variance (MWV) ....................................... 37

Table 2. Geographical variance analysis results for the NDVIc image

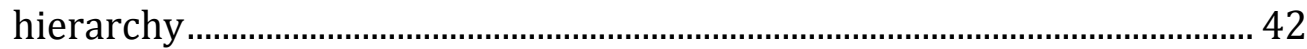




\section{LIST OF FIGURES}

Figure 1. A modified version of Wiens' (1989) figure conceptualizing scale domains, transitions, and thresholds within a hierarchy

Figure 2. Relationships between components of the Definiens Developer 7 (DD7) image segmentation workflow

Figure 3. Example of an irregular, nested hierarchy

Figure 4. Location of Lava Cast Forest (LCF) within Newberry Crater National Volcanic Monument in central Oregon. 25

Figure 5. Rescaled NDIVc image (25 m pixel grain) and analysis extent (1,600 ha) at LCF

Figure 6. Frequency distribution of rescaled NDVIc within the image of LCF

Figure 7. Thematic representation of the rescaled NDVIc image at LCF illustrating the spatial distribution of pixel values corresponding to land-cover types with varying amounts of green vegetation

Figure 8. Box-plots showing the distribution of rescaled NDVIc values with topographic aspect ranges derived from a $10 \mathrm{~m}$ DEM

Figure 9. Normalized Moran's I index and mean weighted variance (MVW) results from the image segmentation optimization analysis at three scale parameters.

Figure 10. The final nested image hierarchy from a portion of the NDVIc image of Lava Cast Forest at incremental scale parameters

Figure 11. Image segmentation parameter combinations evaluated during construction of the NDVIc image hierarchy.

Figure 12. Trends in Moran's I and MWV for image object networks at each level in the image hierarchy. 
Figure 13. Percent change in the number of image objects between

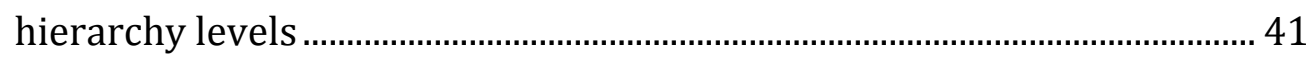

Figure 14. Scale variance and percent sum of squares for each level in the image hierarchy

Figure 15. Landscape patterns at the three hierarchy levels identified by the image segmentation objective function and scale variance analyses..... 44

Figure 16. Two important landscape patterns unrealized in this analysis. 52

Figure 17. A restructuring of Wiens' (1989) figure illustrating the distribution of scale domains, transitions, and thresholds observed in the rescaled NDVIc image of LCF 


\section{INTRODUCTION}

Use of remote sensing and geographic information systems (GIS) for studying landscape patterns demands attention to fundamental issues involving the effects of scale. Specifically, the apparent causes and ecological consequences of emergent patterns depend on the spatiotemporal scales at which ecosystem heterogeneity is measured and evaluated (Turner 1989, Lam and Quattrochi 1992, Levin 1992). Landscape ecologists recognize that no single scale exists at which the complex interactions between biophysical and human forces responsible for generating landscape patterns can be fully explained (Crawley and Harral 2001, Turner 2005). Instead, spatial patterns considered important and predictive at one scale can be equivocal at others. The relative contribution of different soil-forming factors, for instance, appear more or less significant depending on how the soil system is defined and observed (Jenny 1994, McBratney et al. 2003). Furthermore, perceptual biases of spatial heterogeneity differ between species and life history stages (Pribil and Picman 1997). Viewed from an organisimal perspective, habitat considered high quality at a local scale would possess little or no functional value if barriers impeding movement exist or an individual is unable to recognize the area at larger scales (Olden et al. 2004, Schaefer and Mayor 2007). 


\section{Ecosystems as Spatial Hierarchies}

Hierarchy theory as it pertains to ecological systems provides a useful framework for evaluating the effects of scale on observed patterns in landscape heterogeneity. This framework is based on the conceptualization of landscapes as spatial mosaics of patches organized hierarchically across a range of scales ( $\mathrm{O}^{\prime} \mathrm{Neill}$ et al. 1986, Kotliar and Wiens 1990, Wu and Loucks 1995). Patches occurring at each scale level represent discrete landscape features with boundaries constrained by higher level patches and internal structures reflective of the properties and spatial interactions occurring between lower level patches. A hierarchical model's upper and lower levels are predetermined by its specific extent and grain, which limit the possible range of scales over which patterns and processes can be addressed (Wu 1999). Extent refers to the total areal coverage under investigation, while grain corresponds the minimum observable unit. In complex landscapes, changes in scale produce predictable changes in the spatial variance within and between patches with respect to the extent and grain (Wiens 1989). As patch sizes expand within a constant extent, the internal variance increases because a greater proportion of spatial heterogeneity measured at the grain is contained within individual patches. Conversely, differences between patches decrease progressively at higher scales as heterogeneity is consolidated and averaged within larger patches (Wu 1999). 
In order to facilitate a greater understanding of pattern-process relationships using the hierarchical framework, changes in spatial heterogeneity across scales must first be explicitly examined (Wu et al. 2002, Bogaert 2003, Denny et al. 2004). Spatially structured landscapes tend to exhibit characteristic variability over particular ranges of scales (Levin 1992, Wu and David 2002). This property is hypothesized to be the consequence of ecological phenomena operating within different scale domains where observed relationships between spatial patterns and ecosystem processes are either invariant or change monotonically with scale. Sharp transitions in observed patterns or relationships between variables occurring at domain boundaries, referred to as scale thresholds, suggest changes in dominance from one set of pattern-process interactions to another (Wiens 1989) (Figure 1). Positive identification of critical scale domains and thresholds characteristic of different landscapes using remote sensing and GIS may also provide evidence for establishing scaling criteria useful for developing research questions, improving the accuracies of derivative map products, and testing the predictive capacity of ecosystem models (Pickett and Cadenasso 1995, Wu 1999, Wu and Li 2009). Hierarchical models of landscape complexity can either be nested or nonnested. In nested hierarchies, adhering to three rules ensures the result is topologically intact with all patches at one level precisely composed of patches in the next lower level. First, patch boundaries cannot extend beyond the study extent or below the analysis grain. Second, individual patches cannot be contained by more than one patch in the next higher level, although any patch may be composed 
of numerous sub-patches. Third, boundaries of higher-level patches must be consistent with their sub-patches (Burnett and Blaschke 2003). Although nonnested hierarchies are common (e.g., ecological food chains), nested hierarchies are generally considered more suitable for exploratory analysis as details about scale domains and thresholds are more directly obtained by investigating spatial relationships between patches within and across scales (Wu 1999).

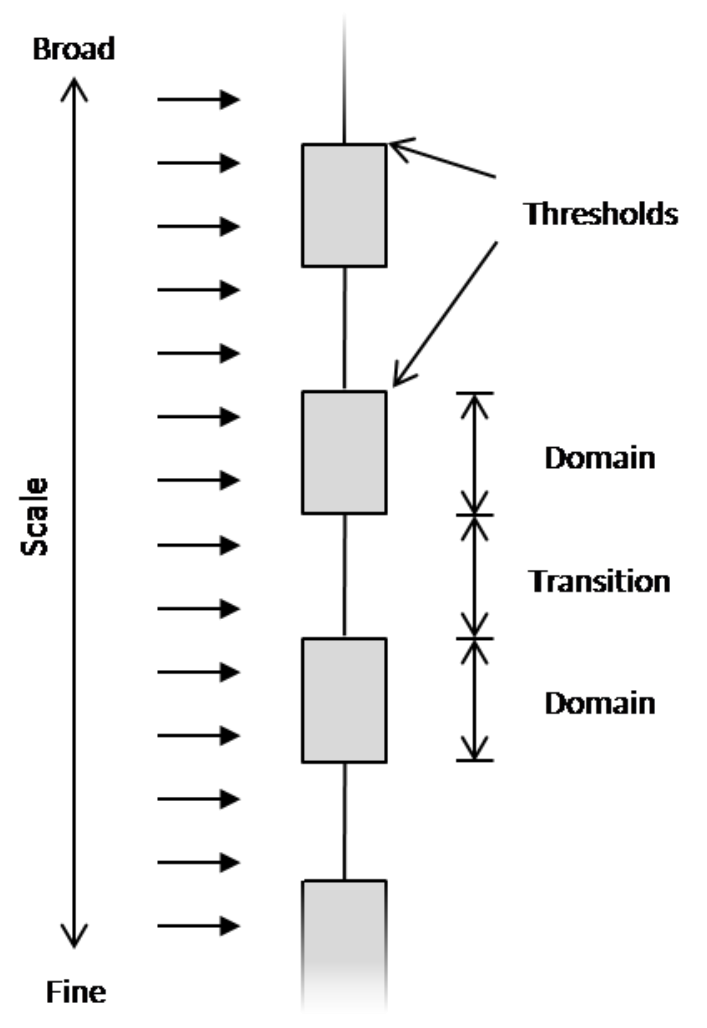

Figure 1. A modified version of Wiens' (1989) figure conceptualizing scale domains, transitions, and thresholds within a hierarchy. Scale domains (grey bars) represent regions of relative stability in pattern-process relationships. Adjacent domains are separated by transitions where ecosystem dynamics appear to behave unpredictably. Thresholds occur at domain boundaries where between-patch differences are maximized and may indicate scales coinciding with dominant landscape patterns. The ability to discern the distribution of domains and transitions depends on the observational scale chosen for an investigation between the extent and grain (i.e., one of the horizontal arrows). 
Two closely related issues, collectively referred to as the modifiable areal unit problem (MAUP), can profoundly bias interpretations of results whenever landscape patches are spatially aggregated (Woodcock and Strahler 1987, Marceau et al. 1994). The first problem is expressed whenever patches are clustered in alternative arrangements at a given scale. The second problem relates to the different inferences obtained whenever patches are increasingly aggregated into larger spatial units (Gotway and Young 2002). Several methods have been proposed to account for the MAUP. For example, aggregations of fine-scale spatial units can be repeated until patches most closely approximate a predetermined target result, such as a particular arrangement of land-cover classes (Openshaw 1977, Hay et al. 2001, Möller et al. 2007, Clinton et al. 2010). This method requires a priori knowledge about the geographic entities of interest and development of a comparative function for determining when results are most satisfactory. Incorporation of spatial statistics designed to deal with the unique qualities of geospatial information can also mitigate the influence of the MAUP. Using the Moran's I and Geary's c statistics, Jelinski and Wu (1996) demonstrated scaling effects related to the MAUP by comparing spatial autocorrelation values for remotely sensed estimates of live vegetation under various aggregation and zoning treatments. Their results revealed differences in the structures of landscape patterns across analysis scales depending on the types and relative complexities of land-cover conditions. This alternative approach to the scale and aggregation problems intentionally recognizes the existence of the MAUP and emphasizes the 
sensitivity of internal and external variations in patch structure to changes in aggregation and zoning rules at different scales (Marceau and Hay 1999). A welldesigned procedure acquiescent to the scope and magnitude of the MAUP can be valuable for discriminating between the potentially large numbers of alternative spatial mosaics of patches and inform construction of hierarchical representations of ecological systems.

Multi-Scale Evaluations of Landscape Patterns with Remotely Sensing

Scaling issues inherent to landscape ecology and addressed by hierarchy theory directly transfer to remote sensing research. As a result, the unique spatiotemporal perspectives of the earth provided by satellite and aerial imagery offer opportunities to evaluate the influences of scale when investigating patterns emergent in complex ecological systems (Wessman 1992, Hay et al. 2002). Images represent arbitrary uniform spatial sampling grids from which measurements of vegetation productivity, canopy structure, topography, and other environmental variables can be reliably obtained (Iverson et al. 1989, Turner et al. 2004, Hilker et al. 2008). Individual pixels with spatial, spectral, and radiometric resolutions predetermined by the acquiring sensor correspond to the minimum observational units (i.e., grain), while the total area of the image scene determines the maximum geographic area available for study (i.e., extent). Hierarchical representations of 
imaged landscapes meeting the nesting criteria are developed using a process known as image segmentation, where neighboring pixels are aggregated into patches, or image objects, at progressively larger scales.

Given the potentially enormous number of different possible pixel and object aggregations, image segmentation ineluctably invokes the MAUP. Consequently, the most valuable segmentation algorithms for evaluating landscape patterns are capable of adequately accounting for spatial heterogeneity in the landscape when decomposing a remotely sensed image into multi-scale representations from a single scale of pixel resolution data, as well as maintaining spatial relationships between objects across all scales. Semantic properties inherited from constituent pixels or sub-objects combined with contextual attributes describing an object's relative position within the horizontal network and vertical hierarchy provide the geospatial information necessary for addressing the MAUP (Burnett and Blaschke 2003). Aggregation issues can then be systematically accounted for by evaluating alternative arrangements of objects when structuring images into a series of nested scales. Subsequent multi-scale analytical techniques based on the hierarchy theory framework can be applied to assist in defining dominant, emergent patterns relevant to ecological processes within complex landscapes. 


\section{Research Objectives}

In this paper, I focus on evaluating the multi-scale structure of landscape patterns using remote sensing, and not on patterns as a cause or consequence of one or more ecological processes. By acknowledging the effects of scale on measurements of spatial heterogeneity within and across scales, I address two fundamental challenges confronted early in investigations of landscape pattern using image segmentation and hierarchical image analysis:

1. Characterization of alternative arrangements of image objects to inform selection of image segmentation parameters

2. Identification of dominant, multi-scale patterns within a nested image hierarchy potentially important for informing subsequent geospatial and ecological analyses.

Both issues can introduce research biases that can affect subsequent data interpretations and applications. Image objects produced with arbitrarily chosen segmentation parameters possess questionable explanatory power, are often not reproducible, and can precipitate misleading conclusions. Furthermore, the scale of objects deemed appropriate for one study may not be transferable to another because of differences in an image's representation of the landscape, the specific research questions being asked, and the methods used to extract information from the imagery (Woodcock and Strahler 1987). 
In the following sections, I first describe the motivations and procedures for segmenting imagery and constructing image hierarchies using the algorithm implemented in Definiens Developer 7 (DD7) software (Definiens 2007). I then present two analytical methods for addressing issues related to segmentation parameterization, image hierarchy development, and the MAUP. In order to be applicable in a wide-range of situations, these methods incorporate established geostatistical techniques impartial to most types of input geospatial data and amendable to various image analysis and GIS software. The first method enlists an objective function for testing how small parameter adjustments influence aggregation results, and provides a rational for selecting one combination of parameters over another. The second method employs a hierarchical image analysis technique capable of detecting and defining distributions of scale domains and thresholds. The usefulness of these methods is demonstrated using a satellite image transformed to portray variations in vegetation cover density within a complex forested landscape in central Oregon. Results from the application of both methods are then discussed in terms of their utility and capacity to provide insight into the effects of scale in ecologically-oriented remote sensing applications. 


\section{METHODS}

\section{Image Segmentation}

The typical motivation for image segmentation in remote sensing is to change a scene's spatial structure from a gridded pixel array into a mosaic of image objects more closely approximating real-world entities. Achieving high quality segmentations for a given application is critical since resulting objects and their patterns comprise the foundation data set for subsequent analyses. Several factors make this a particularly challenging task. Foremost among these challenges are the acquiring sensor's spatial, spectral, and radiometric resolutions that predetermine the minimum observation scale, possible ancillary images (e.g., vegetation indices), and measurement precision (Woodcock and Strahler 1987). Environmental conditions at the time of collection also influence image properties and, consequently, object discrimination and segmentation quality (Benz et al. 2004).

Several segmentation procedures with varying levels of sophistication and suitability for specific image types are available including graph partitioning and neural network segmentations (Pal and Pal 1993). The technique available in the Definiens Developer 7 (DD7) software used for this research belongs to a class of sequential agglomerative algorithms that cluster pixels based on their similarity to a test statistic calculated from user-defined criteria and image information (Legrende and Legrende 1988, Burnett and Blaschke 2003, Flanders et al. 2003). This 
approach merges neighboring pixels according to the combined influence of two heterogeneity criteria: color and shape (Figure 2). Color refers to an input image's digital values and is the principle information used to compare adjacent pixels. Weights assigned to each input image are used to allocate their percent contribution to the color criteria. Shape adjusts image object geometry in terms of smoothness and compactness. Higher smoothness values reduce fraying along image object borders while higher compactness values control for convoluted edges in highcontrast regions of the image. Parameter values between 0.0 and 1.0 are specified by the analyst for compactness and shape, and are used by the software to calculate smoothness and color. Neighboring pixels are aggregated until the standard deviation of a candidate region's heterogeneity criteria is less than the usersupplied scale parameter. The operation proceeds until all pixels in the image have been evaluated against their neighbors, and a continuous network of objects is generated (Benz et al. 2004, Esch et al. 2008). The ability to generate alternative arrangements of image objects by adjusting parameters and create horizontally and vertically integrated image hierarchies by successively merging regions generated in preceding levels makes the DD7 approach suitable for evaluating the effects of scale in remote sensing investigations of landscape spatial patterns. 


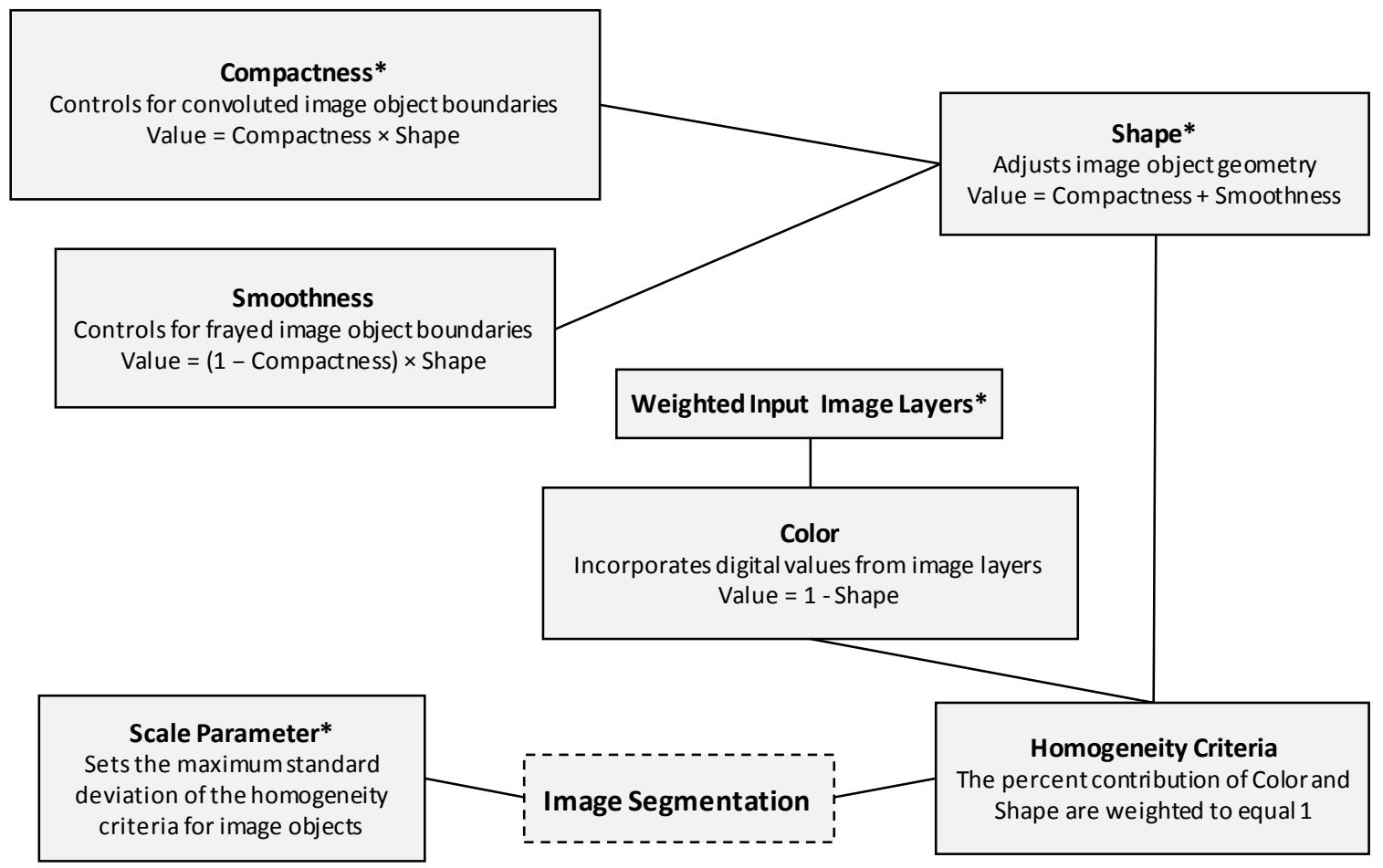

Figure 2. Relationships between components of the Definiens Developer 7 (DD7) image segmentation workflow. The required user-input parameters are designated with asterisks.

Region-merging in DD7 is founded on the relationship between the heterogeneity criteria and scale parameter. Higher scale parameters allow regions to possess higher levels of internal variability. As a result, increasing the scale parameter cannot split apart an existing image object, but will merge it with one of its neighbors or carry it forward intact to the next higher level. This property has two important consequences for how scale is perceived. First, within-object variance increases as regions become larger, diminishing the likelihood of heterogeneity criteria falling below higher scale parameter thresholds. This effect is 
evident by a rapid decrease in the number of merging events occurring between higher hierarchy levels. Second, the scale parameter imposes a discontinuous structure onto the imagery. Unlike a regular nested hierarchy where each level represents a uniform clustering of sub-level objects (e.g., image block statistics), an individual image object can potentially persist across multiple levels, and a single areal unit cannot be used to quantify the changes in measurement scale. Instead, each object is considered a discrete entity occurring at a specific observation scale within the integrated image hierarchy (Legrende and Legrende 1988).

The need for a systematic approach to image segmentation is realized when every potential combination of parameters can theoretically produce a unique result, and subjectively choosing the between spatial mosaics with trial-and-error methods can become time-consuming and delusive. Existing methods for addressing segmentation quality focus on how well image objects agree with existing reference polygons or conform with expected classification results (Zhang 1996, Clinton et al. 2010). These solutions, however, are often non-transferable, spatially bounded, and ineffective without preconceptions about the identities and geographic scales of scene elements. Ancillary reference information may also represent definitional rather than operational landscape designations, with the former being potentially less conducive to assessments of ecological processes using hierarchy analysis (Wu 1999). For example, restricting image segmentations to a specific land ownership designation may mask important fine-scale patterns by imposing unjustifiable higher-level constraints and contexts. Furthermore, any 
errors and biases contained in reference data sets are likely to affect segmentation selection and be carried forward to future applications. Approaching image segmentation without presumptions about the best landscape decomposition is often preferable since the observational units most closely related to system processes are allowed to emerge objectively (O’Neill et al. 1986). This requires optimal segmentation results to be realized at different observation scales directly from analysis of the source imagery.

\section{An Objective Function for Evaluating Segmentation Quality}

Accepting any image segmentation result invites problems whenever spatial information is aggregated to create meaningful analysis units (i.e., the MAUP). An empirical methodology designed to facilitate discrimination between alternative image segmentations based exclusively on sensor measurements requires a clear definition of what constitutes a most satisfactory solution. Objective functions reflecting the conditions of alternative segmentations can facilitate the decisionmaking process in situations where a standard criterion for high quality groupings can be established (Ward 1963). In this research, the primary goal of image segmentation is to differentiate homogeneous regions within a landscape, and the most satisfactory results are considered to occur when differences between objects are greatest and variability within objects is minimized. Using established statistical 
techniques, Espindola et al. (2006) presented an objective function for evaluating these two conditions and selecting among various combinations of segmentation parameters. The function incorporates two indices with opposing gradients.

The first index expresses the degree of similarity between objects within the entire segmentation network based on the Moran's I spatial autocorrelation index. Spatial autocorrelation is a well-documented property of spatial data including remotely sensed imagery, and is present when values for a variable are spatially dependent (Marceau et al. 1994, Ord and Getis 1995). Moran's I index is calculated by:

$$
I=\frac{n \sum_{i}^{n} \sum_{j}^{n} w_{i j}\left(y_{i}-\bar{y}\right)\left(y_{j}-\bar{y}\right)}{\left(\sum_{i}^{n} \sum_{j}^{n} w_{i j}\right) \sum_{i}^{n}\left(y_{i}-\bar{y}\right)^{2}}
$$

where there are $n$ image objects, $y_{i}$ and $y_{j}$ are the mean pixel values of objects $i$ and $i$, and $\bar{y}$ is the mean of the entire image. $w_{i j}$ is a measure of spatial proximity, and is equal to 1 if objects $i$ and $j$ are spatially adjacent, otherwise $w_{i j}$ equals 0 (Rogerson 2001). Moran's I index values range from -1 to +1 , with results near +1 indicating a higher degree of similarity or high spatial autocorrelation between neighboring objects. Values approaching 0.0 indicate lower spatial autocorrelation and greater distinction between objects in the segmented network. 
The objective function's second index expresses within-object variability based on the mean weighted variance (MWV) defined as the sum of all products of variance and area divided by the sum of all areas:

$$
M W V=\frac{\sum_{i}^{n} a_{i} \times v_{i}}{\sum_{i}^{n} a_{i}}
$$

where $a_{i}$ is the area of object $i$ and $v_{i}$ is its internal variance (Espindola et al. 2006). By weighting objects by their area, more emphasis is placed on larger regions with inherently greater interior heterogeneity.

The Moran's I index and MWV are first normalized before being incorporated into the final object function given by:

$$
\begin{gathered}
F(v, I)=F(v)+F(I) \\
\text { where } F(X)=\frac{X_{\max }-X}{X_{\max }-X_{\min }}
\end{gathered}
$$

The optimal image segmentation is considered the result generated by the combination of heterogeneity criteria leading to the highest object function value at a given scale parameter. Once identified, the object network becomes one level in the developing hierarchy. The optimization process is then repeated to produce the next higher level by applying the DD7 segmentation algorithm using the next higher scale parameter. Ensuring all segmentation parameter settings in increments small enough to ensure potentially important image object configurations is critical. The method should also be as automated as possible to efficiently address the large numbers of possible image segmentations produced. 
The image segmentation optimization approach described by Espindola et al. (2006) provides a methodology for objectively restructuring a remotely sensed image into a multi-scaled hierarchy of image objects. It does not, however, accommodate the detection of appropriate scales for observing spatial patterns relevant to ecological processes operating within a landscape. Organizing an image hierarchically with successive image segmentations does not imply that the environmental variables under investigation are actually ordered in a nested series of spatial scales. Additional techniques must be used to detect structured spatial variation at the scales over which different processes may be occurring (Bellehumeur and Legendre 1998).

\section{Geographic Variance Analysis}

The geographic variance method proposed by Moellering and Tobler (1972) is a hierarchical analysis that determines the relative variability and independent contribution to the total variance of each level in a nested hierarchy. The method partitions a hierarchy's total variability using the sum of squares at each level. According to the authors, levels exhibiting peaks in the scale-variance ratio correspond to the aggregation levels where spatial patterns and processes are most likely to be operating and should be considered for subsequent analysis. Geographic variance analysis remains generally untested in remote sensing (Wu and $\mathrm{Li}$ 2009). 
One likely reason is the recent introduction of image segmentation techniques capable of efficiently generating and evaluating irregularly nested hierarchies. The geographic variance method represents a potentially robust approach to characterizing relationships between landscape patterns and scale in remote sensing, but its reliability remains unclear and warrants systematic investigation (Cao and Lam 1997, Stoms 1994, Wu 1999). This argument is supported by Wu et al. (2000), who concluded the geographic variance method possessed a greater capacity for discerning multi-scale spatial patterns in artificially constructed landscapes over more traditional semivariogram based analyses. A careful accounting for the influence of the MAUP during hierarchy construction, however, remains prerequisite as the technique can be applied to any nested hierarchy and does not directly address either the aggregation or scaling issues.

Using the example four-level hierarchy in Figure 3, the geographic variance statistical model is expressed as:

$$
\mathrm{X}_{i j k}=\mu+\alpha_{i}+\beta_{i j}+\gamma_{i j k}
$$

where $\mathrm{X}_{i j k}$ corresponds to the value of a spatial unit (e.g., an image object or pixel) at the smallest-scale hierarchy level. The grand mean of the hierarchy is defined as:

$$
\mu=\frac{\sum_{i} \sum_{j} \sum_{k} \mathrm{X}_{i j k}}{N}=\overline{\mathrm{X}}_{\ldots}
$$

where $N$ is the total number of $\mathrm{X}_{i j k}$ observations within hierarchy's spatial extent (Level 3 objects within Level 0 in Figure 3 ) and $\overline{\mathrm{X}}$.. indicates that $\mu$ is the mean of $\mathrm{X}$ summed over the subscripts represented by dots (see matching notation in Figure 
3). The remaining model terms are the effects at the different hierarchy levels, with the $\alpha$ level corresponding to the second highest hierarchy level (Level 1 in Figure 3) preceded by the $\beta$ and $\gamma$ levels underneath (Levels 2 and 3 in Figure 3, respectively). The effect of the $\alpha$ level is calculated by:

$$
\begin{aligned}
& \qquad \alpha_{i}=\overline{\mathrm{X}}_{i .}-\overline{\mathrm{X}}_{\ldots} \\
& \text { where } \overline{\mathrm{X}}_{i .}=\frac{\sum_{j} \sum_{k} \mathrm{X}_{i j k}}{n_{i}}
\end{aligned}
$$

and $n_{i}$ is the number of areas in the $i^{t / \hbar}$ region. The effect of the $\beta$ level is:

$$
\begin{gathered}
\qquad \beta_{i j}=\overline{\mathrm{X}}_{i j,}-\overline{\mathrm{X}}_{i, \mathrm{~s}} \\
\text { where } \overline{\mathrm{X}}_{i j .}=\frac{\sum_{k} \mathrm{X}_{i j k}}{n_{i j}}
\end{gathered}
$$

and $n_{i j}$ is the number of areas in the $j^{t / h}$ aggregation of the $i^{t / 4}$ region. Finally, the effect of the lowest hierarchy level is:

$$
\gamma_{i j k}=\mathrm{X}_{i j k}-\overline{\mathrm{X}}_{i j}
$$

Rewriting the scale variance model and substituting for $\mu, \alpha_{i}, \beta_{i j}$, and $\gamma_{i j k}$ produces:

$$
\begin{gathered}
\mathrm{X}_{i j k}-\mu=\alpha_{i}+\beta_{i j}+\gamma_{i j k} \\
\mathrm{X}_{i j k}-\overline{\mathrm{X}}_{\ldots}=\left(\overline{\mathrm{X}}_{i \mathrm{~m}}-\overline{\mathrm{X}}_{\ldots}\right)+\left(\overline{\mathrm{X}}_{i j .}-\overline{\mathrm{X}}_{i \mathrm{~s}}\right)+\left(\mathrm{X}_{i j k}-\overline{\mathrm{X}}_{i j .}\right)
\end{gathered}
$$


Squaring and summing over all subscripts leads to the final scale variance equation:

$$
\begin{aligned}
\sum_{i=1}^{I} \sum_{j=1}^{J_{i}} \sum_{k=1}^{K_{i j}}\left(\mathrm{x}_{i j k}\right. & \left.-\overline{\mathrm{X}}_{\ldots}\right)^{2} \\
= & \sum_{i=1}^{I} \sum_{j=1}^{J_{i}} \sum_{k=1}^{K_{i j}}\left(\overline{\mathrm{X}}_{i . .}-\overline{\mathrm{X}}_{\ldots}\right)^{2}+\sum_{i=1}^{I} \sum_{j=1}^{J_{i}} \sum_{k=1}^{K_{i j}}\left(\overline{\mathrm{X}}_{i j .}-\overline{\mathrm{X}}_{i . .}\right)^{2} \\
& +\sum_{i=1}^{I} \sum_{j=1}^{J_{i}} \sum_{k=1}^{K_{i j}}\left(\mathrm{x}_{i j k}-\overline{\mathrm{X}}_{i j .}\right)^{2}
\end{aligned}
$$

which represents a partitioned expression of each level's relative contribution to the hierarchy's total variation in terms of the total sum of squares:

$$
S S_{\text {Total }}=S S_{\alpha}+S S_{\beta}+S S_{\gamma}
$$

The hierarchy shown in Figure 3 represents an example of an "irregular case" described by Moellering and Tobler (1972). In these situations, the hierarchy is unevenly nested such that all objects at a given level do not contain the same number of sub-objects. The expected mean square (EMS) of each level in an 
irregularly nested hierarchy is calculated by dividing the sums of the squared deviations by the appropriate degrees of freedom:

$$
\begin{gathered}
E M S_{\alpha}=\frac{\sum_{i} n_{i} \alpha_{i j}^{2}}{I-1} \\
E M S_{\beta}=\frac{\sum_{i} \sum_{j} n_{i j} \beta_{i j}^{2}}{\sum_{i}\left(J_{i}-1\right)} \\
E M S_{\gamma}=\frac{\sum_{i} \sum_{j} \sum_{k} n_{i j k} \gamma_{i j k}^{2}}{\sum_{i} \sum_{j}\left(K_{i j}-1\right)}
\end{gathered}
$$

The scale-variance (SV) components for each level are given by:

$$
\begin{gathered}
S V_{\alpha}=\frac{\sum_{i}^{I} \alpha_{i}^{2}}{I-1} \\
S V_{\beta}=\frac{\sum_{i}^{I} \sum_{j}^{J_{i}} \beta^{2}{ }_{i j}}{\sum_{j}\left(J_{i}-1\right)} \\
S V_{y}=\frac{\sum_{i}^{I} \sum_{j}^{J_{i}} \sum_{k}^{K_{i j}} \gamma_{i j k}^{2}}{\sum_{j} \sum_{k}\left(K_{i j}-1\right)}
\end{gathered}
$$

Results of the geographic variance method are typically presented as barcharts or histograms depicting the scale-variance components and percent sum of squares for each hierarchy level. Peaks in scale-variance identify scales where the structure of landscape patterns generated by the environmental variable being studied exhibits the greatest variability. The magnitude of percent sum of square bars reflect the relative contribution of each level to the total landscape variability (Moellering and Tobler 1972). 
Level 0
Largest Scale

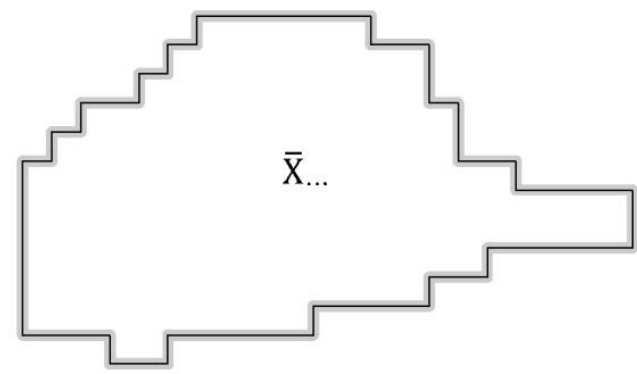

Level 1

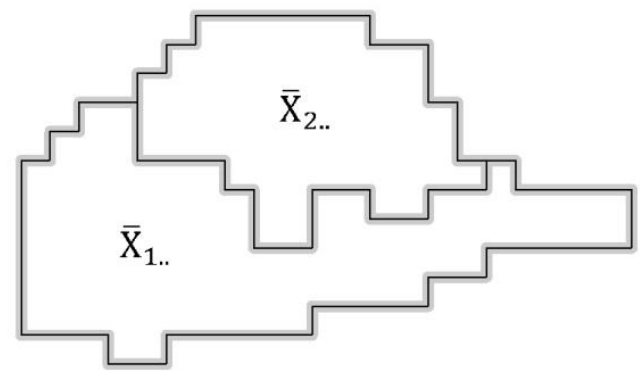

Level 2

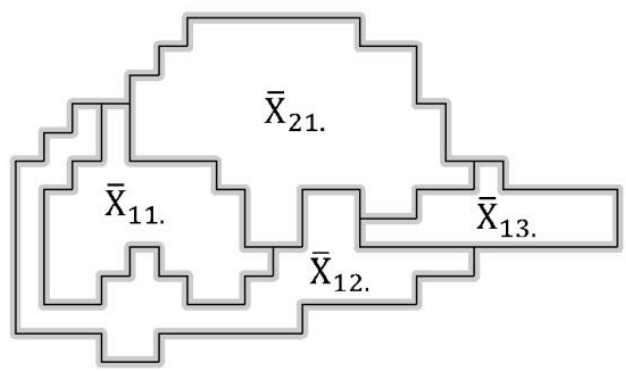

Level 3

Smallest Scale

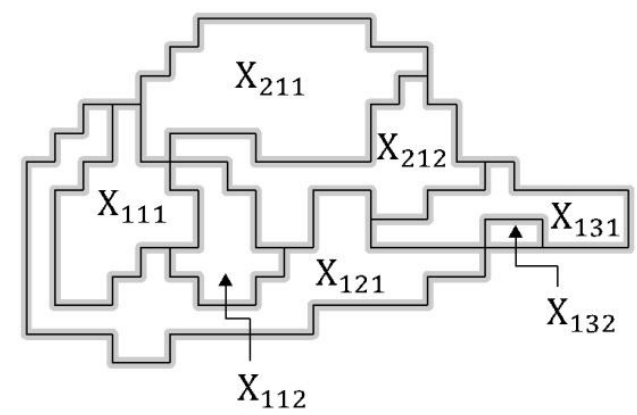

Figure 3. Example of an irregular, nested hierarchy. The notation used to designate each object (e.g., $\overline{\mathrm{X}}_{w}, \overline{\mathrm{X}}_{1_{m}}, \overline{\mathrm{X}}_{12}$, and $\overline{\mathrm{X}}_{123}$ ) is described in the text. 


\section{Study Area}

My research area is the heterogeneous landscape at Lava Cast Forest (LCF) located within Newberry Crater National Volcanic Monument and the Deschutes National Forest in central Oregon (Figure 4). LCF is characterized by 12 forested cinder cones (kipukas) isolated from the surrounding forest within barren, midHolocene lava flows (Peterson and Groh 1969). Kipukas range in size from 0.4 to 113 ha. Elevations within the study area range between approximately 1,550 and $1,900 \mathrm{~m}$.

A rain shadow created by the Cascade Range west of LCF creates semi-arid climate conditions and strongly influence the forest vegetation in the study area. Seasonal precipitation, 35 to $70 \mathrm{~cm}$ annually, falls predominantly as snow during winter and rain in summer convective storms (Franklin and Dryness 1988). Three topographically and edaphically distributed tree species dominate the kipukas and surrounding forest (Pohl et al. 2006). A white fir-grand fir hybrid (Abies concolor A. grandis) occurs most frequently on north-facing slopes and interior stands, while ponderosa pine (Pinus ponderosa) dominates on south-facing slopes. Level areas are commonly covered by dense stands of lodgepole pine (P. contorta).

The heterogeneous landscape at LCF is largely the result of a dynamic disturbance history. Portions of three lava flows occurred between 5,800 and 6,400 years BP dissect the area (Jensen 2000) and create edges that influence forest composition and structure (Hrinkevich 2005). Fire history reconstructions by 
Arabas et al. (2006) identified 98 fire events over an 800 year period with mean fire return intervals dependent on local topography, soils, and the spatial arrangement of kipukas relative to the surrounding forest. Fires on the kipukas were historically smaller and patchy compared to those in the surrounding forest. Three large fires spread across the study area in 1740,1823 , and 1918. A mixed-severity blow-down significantly altered canopy conditions on the eastern slope of the largest southern kipuka within the past 30 years (Karps 2006). Spatially variable distributions and densities of two dwarf mistletoe species infect ponderosa pine and lodgepole pine stands exist throughout the kipukas (Stanton and Arabas 2009). Active timber management continues within the surrounding forest and occurred on Hoffmann Island, the northern most kipuka as recently as the 1960s. Restricted access has prevented harvesting on all other kipukas, maintaining them in relatively pristine condition. 


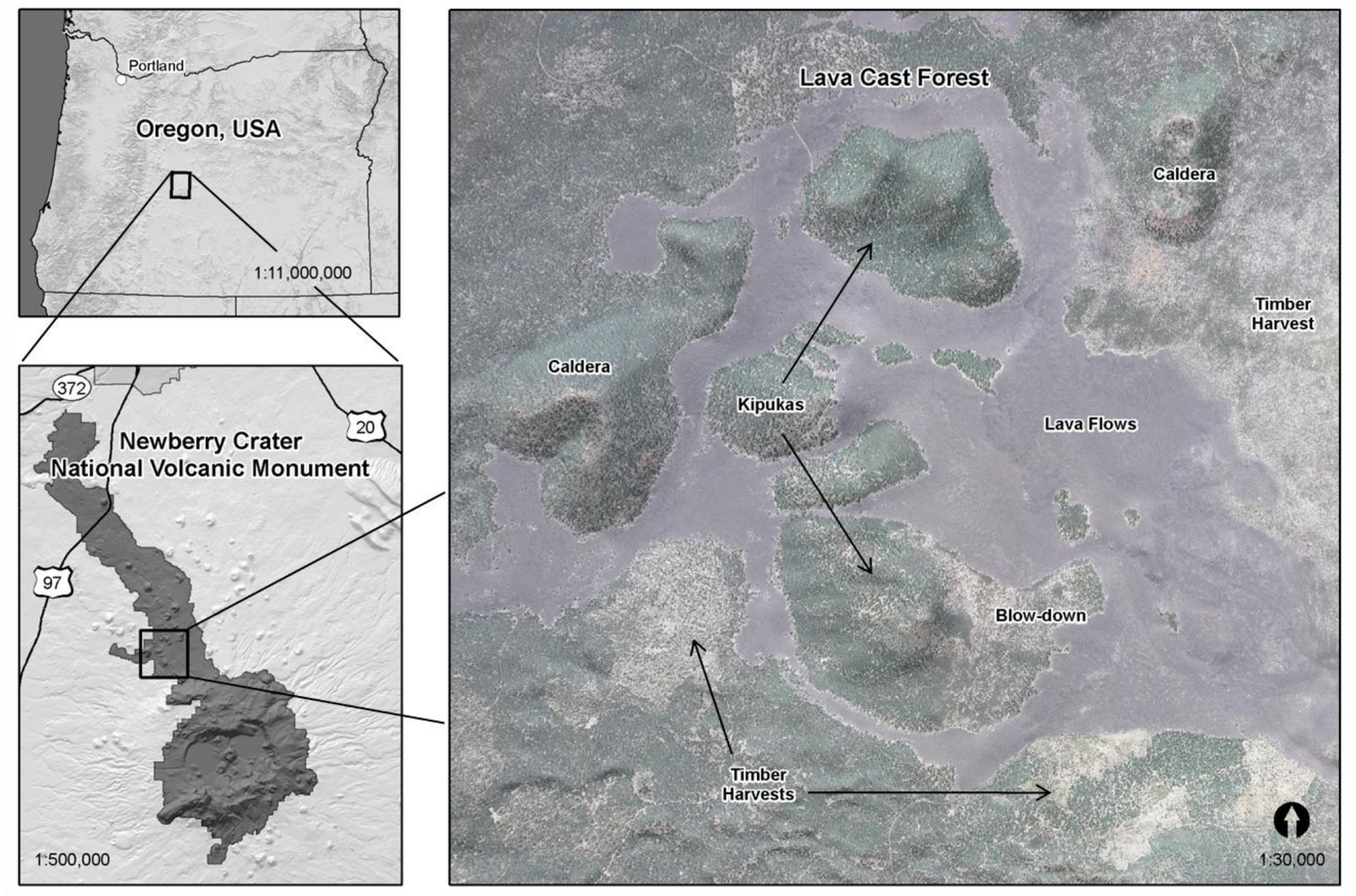

Figure 4. Location of Lava Cast Forest (LCF) within Newberry Crater National Volcanic Monument in central Oregon. Landscape features characterizing the study area are identified on a 2005 NAIP aerial photograph. 
Image Processing

Reflective properties of vegetation at different regions of the electromagnetic spectrum are commonly used to generate proxy estimates of net primary productivity and other environmental variables for analyzing spatial heterogeneity (Gamon et al. 1995, Riera et al. 1998, Todd et al. 1998, Ustin et al. 2004). This research incorporates a middle-infrared corrected version of the normalized difference vegetation index (NDVIc) derived from a $25 \mathrm{~m}$, Landsat ETM+ satellite image collected on 17 June 2004 to assess the relative amount and conditions of forest vegetation cover within the rectangular extent of a $2 \mathrm{~km}$ radius circle centered on LCF (Figure 5). NDVIc adjusts background reflectance values to strengthen predictions of plant area index in complex terrain and semi-open to sparse canopies (Walsh et al. 1997, Pocewicz et al. 2004). The expression for NDVIc is:

$$
\begin{aligned}
N D V I C= & (N I R-R E D) /(N I R+R E D) \\
& \times\left[1-\left(M I R-M I R_{\min }\right) /\left(M I R_{\max }-M I R_{\min }\right)\right]
\end{aligned}
$$

where NIR corresponds to the Landsat ETM+ near-infrared band (channel 4), RED to the visible red band (channel 3), and MIR to one of the two middle-infrared bands (channels 5 or 7). I added a constant value of 0.1 to each band before calculating NDVIc to avoid dividing by zero. NDVIc values range from -1.0 to 1.0 , with more positive values signifying higher densities of green vegetation. I rescaled NDVIc 
values between 0 and 200 prior to analysis to improve computer visualization using the formula:

$$
\text { Rescaled NDVIc }=(\text { NDVIC }+1) \times 100
$$

Associations between rescaled NDVIc values and dominate land-cover types based on site visits and previous research (Hadley and Arabas in prep) were derived using visual interpretation of the image and its pixel frequency distribution. I summarized rescaled NDVIc values by topographic aspect derived from a $10 \mathrm{~m}$ USGS digital elevation model (DEM) using spatial analysis tools available in ArcGIS 10 Beta (ESRI 2010).

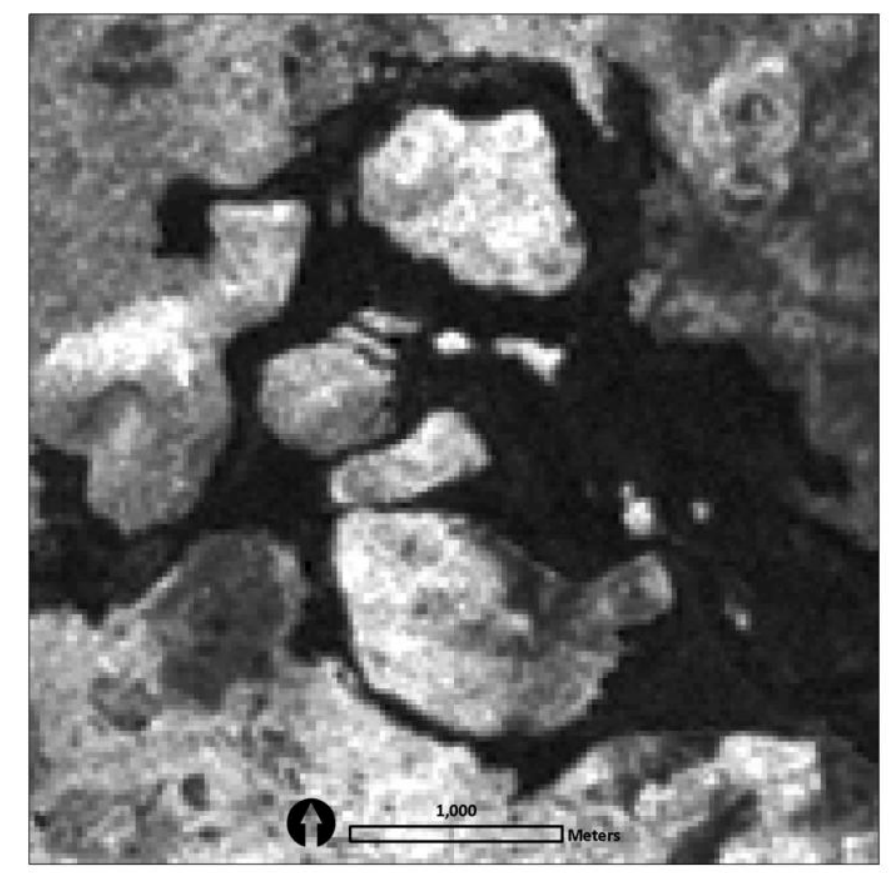

Figure 5. Rescaled NDIVc image (25 m pixel grain) and analysis extent (1,600 ha) at LCF. Higher NDVIc values have brighter grey-tones, and indicate a greater presence of green vegetation. 
Image Segmentation and Hierarchical Image Analysis

I organized the NDVIc image of LCF into a nested hierarchy of image objects by successively applying the DD7 segmentation algorithm at incrementally larger scale parameters. Image pixels were considered objects in the lowest hierarchy level (i.e., scale parameter $=0$ ). Objects at each higher level corresponded to the optimal clustering of the previous level's objects chosen from alternative combinations of heterogeneity criteria at a single scale parameter using the objective function described by Espindola et al. (2006).

Beginning with a scale parameter of 2 , image pixels were aggregated twenty times using compactness parameter $0.1,0.3,0.5,0.7$, and 0.9 paired with shape parameters $0.1,0.3,0.5$, and 0.7 . Segmentations were exported as vector polygons attributed with the scale parameter, pixel mean, pixel variance, and object area. I used Python scripting to automate the calculation of MWV and global Moran's I index using geoprocessing tools available in ESRI ArcGIS 10 Beta. Spatial autocorrelation calculations were facilitated using spatial weights matrices with Euclidean distances and connectivity conceptualized as objects sharing a boundary and/or a node. Normalization of each component and calculation of the final objective function values were performed manually. The image segmentation parameter set producing the highest objective function value was used to generate the first hierarchy level containing pixel aggregations. I repeated the entire process to generate each higher level in the hierarchy using scale parameter increments of 2. 
Whenever multiple combinations of parameters produced identical segmentation results, I used the lowest compactness and shape parameters. I accounted for sample size limitations in the calculation of the Moran's I statistic by terminating hierarchy development when the number of objects comprising the highest partitioned hierarchy level was less than 30 objects. The remaining objects were merged into a single object. The final result was an image hierarchy bounded by pixels as objects in the lowest level and the image extent at the highest level where each intermediate level contained clusters of the previous level's objects exhibiting the highest objective function value.

Following construction of the final NDVIc image hierarchy, all image objects were exported from DD7 as vector polygons attributed with the aggregate mean, the mean value of the object one level above, the number of sub-objects one level below, and the total number of constituent pixels. Using this information, I applied the set of equations outlined by the geographic variance analysis method (Moellering and Tobler 1972) to the final hierarchy using Python scripting. 


\section{RESULTS}

Pixel-Level Patterns in NDVIC

The frequency distribution of rescaled NDVIc values at Lava Cast Forest (LCF) ranges between 91.3 and 153.2 with three maxima apparent at bins 100, 108, and 121 (Figure 6). Field observations and visual interpretation of a thematic representation of the image partitioned at the minimum values on either side of these peaks correspond to the three dominate landscape cover types in the scene (Figure 7). Values associated with the lowest peak comprise approximately $31 \%$ of all image pixels, and coincide with the lava flows and isolated areas of barren ground. The majority of pixels associated with the middle peak correspond with either lava/forest edge conditions, recently disturbed areas caused by timber harvests in the surrounding forest, and blow-down on the most southern kipuka. The third peak incorporates the widest range of rescaled NDVIc values, and equates to forest vegetation in the remainder of the study area (Figures 4 and 7). Values for these pixels are highest on north and west-facing slopes and the lowest on southern aspects (Figure 8).

Each of the 12 kipukas at LCF is discernable in the $25 \mathrm{~m}$ pixel grain NDVIc image; the smallest, located on the western edge of the most northern kipuka, is composed of approximately four pixels. Variations in rescaled NDVIc summarized by topographic aspect reveal the presence of open calderas in the northeastern and 
west central portions of the image. Patterns within the lava fields appear oriented with flow direction, and are higher in areas with minimal vegetation cover.

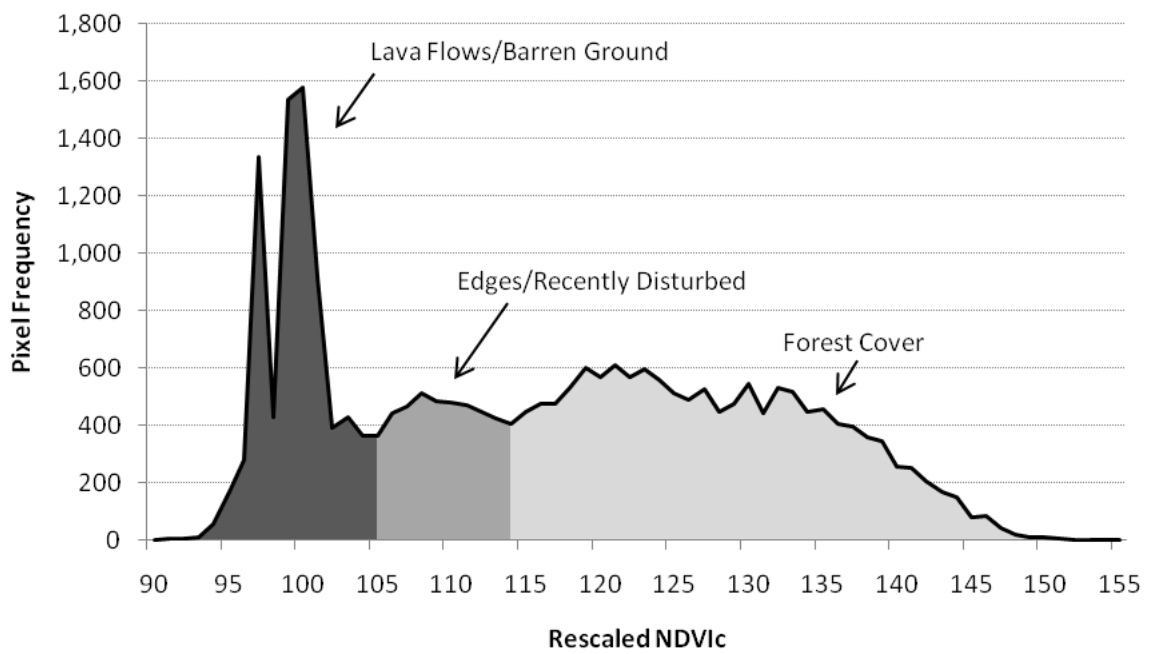

Figure 6. Frequency distribution of rescaled NDVIc within the image of LCF. Ranges of pixel values associated with different land-cover types are demarcated with progressively lighter grey-tones. 


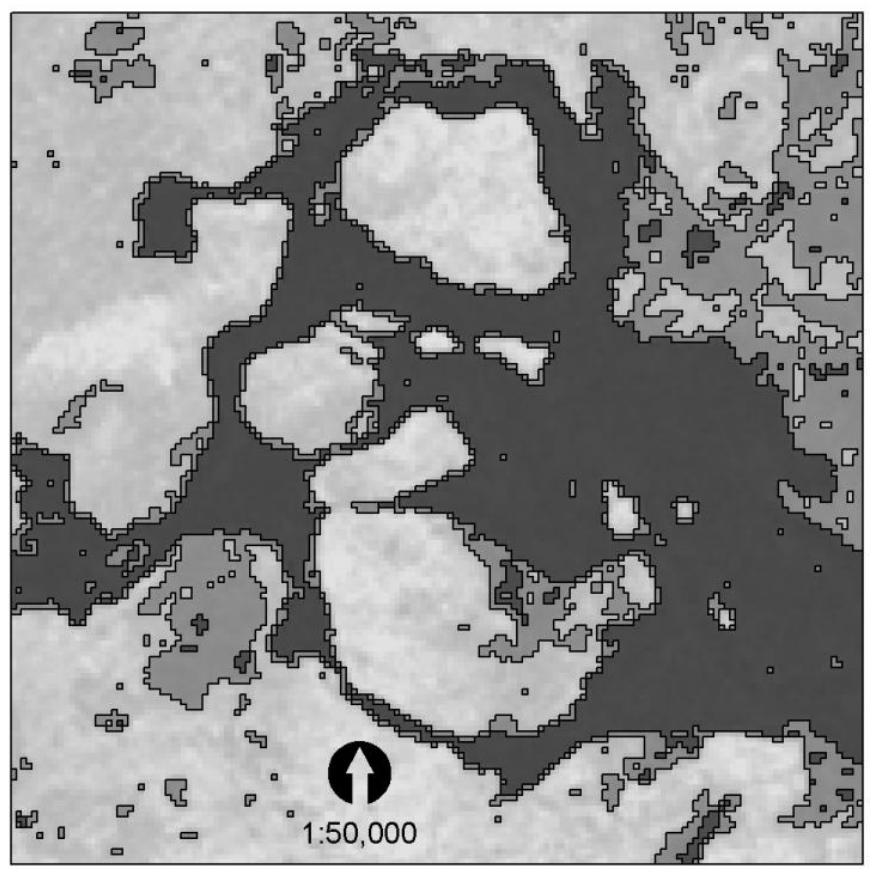

Figure 7. Thematic representation of the rescaled NDVIc image at LCF illustrating the spatial distribution of pixel values corresponding to land-cover types with varying amounts of green vegetation. Grey-tones match those shown in Figure 6.

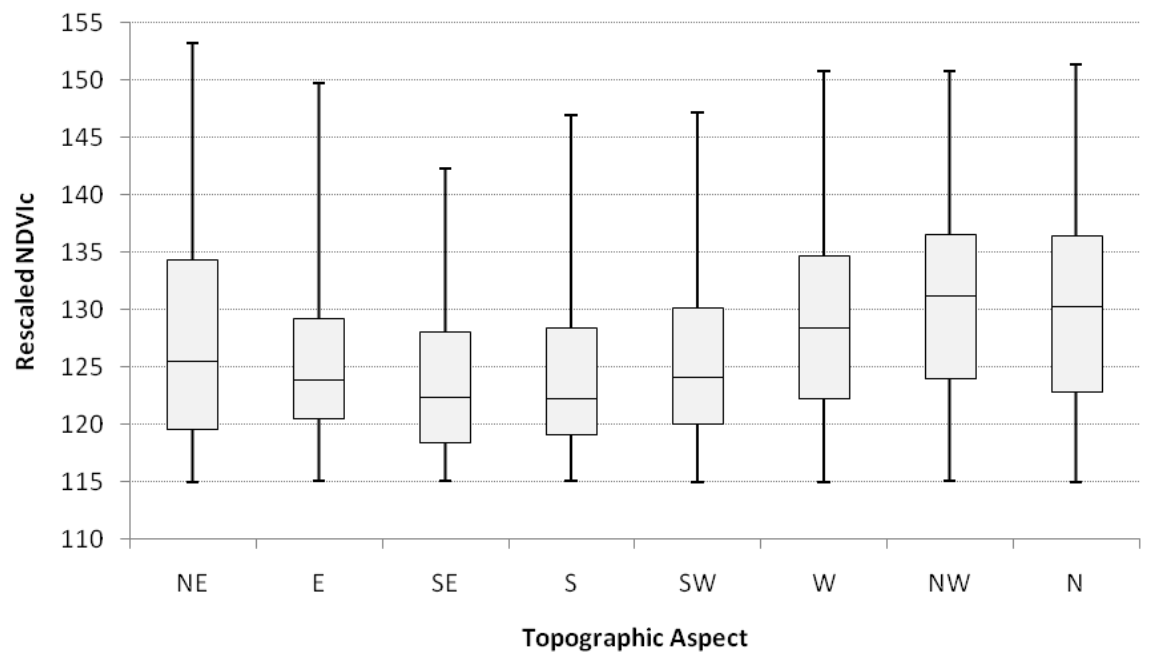

Figure 8. Box-plots showing the distribution of rescaled NDVIc values with topographic aspect ranges derived from a $10 \mathrm{~m}$ DEM. 
The Effects of Alternative Parameters on Segmentation Results

Evaluations of alternative combinations of compactness and shape parameters using normalized Moran's I and mean weighted variance (MWV) yielded a wide range of results (Figure 9). At each scale parameter, normalized Moran's I values vary unpredictably with compactness and shape. Normalized MWV values, however, exhibit similar trends between scale parameters. Initially, the full range of compactness parameters consistently produce image object networks with the lowest average within-object heterogeneity at lower shape values. This trend is interrupted at higher shape values by a sharp decline in normalized MWV with increasing compactness.

The final NDVIc image hierarchy consists of 14 nested levels, beginning with the image pixels as objects in the lowest level (scale parameter $=0)($ Figure 10). Image object grey tones reflect the average NDVIc value within each object relative to the entire scene. Aggregation events occur most frequently in the first five levels, after which objects are more likely to persist through two or more levels. Object boundaries in lower levels coincide with local variations in land cover and segregate edge conditions between contrasting cover types (e.g., lava and forest). Edge objects eventually incorporate into one of their neighboring land cover types at higher levels. Patterns in vegetation cover related to topographic aspect and recent disturbances identified in the image emerge at scale parameter 10 and are clearly recognizable by scale parameter 18 . 

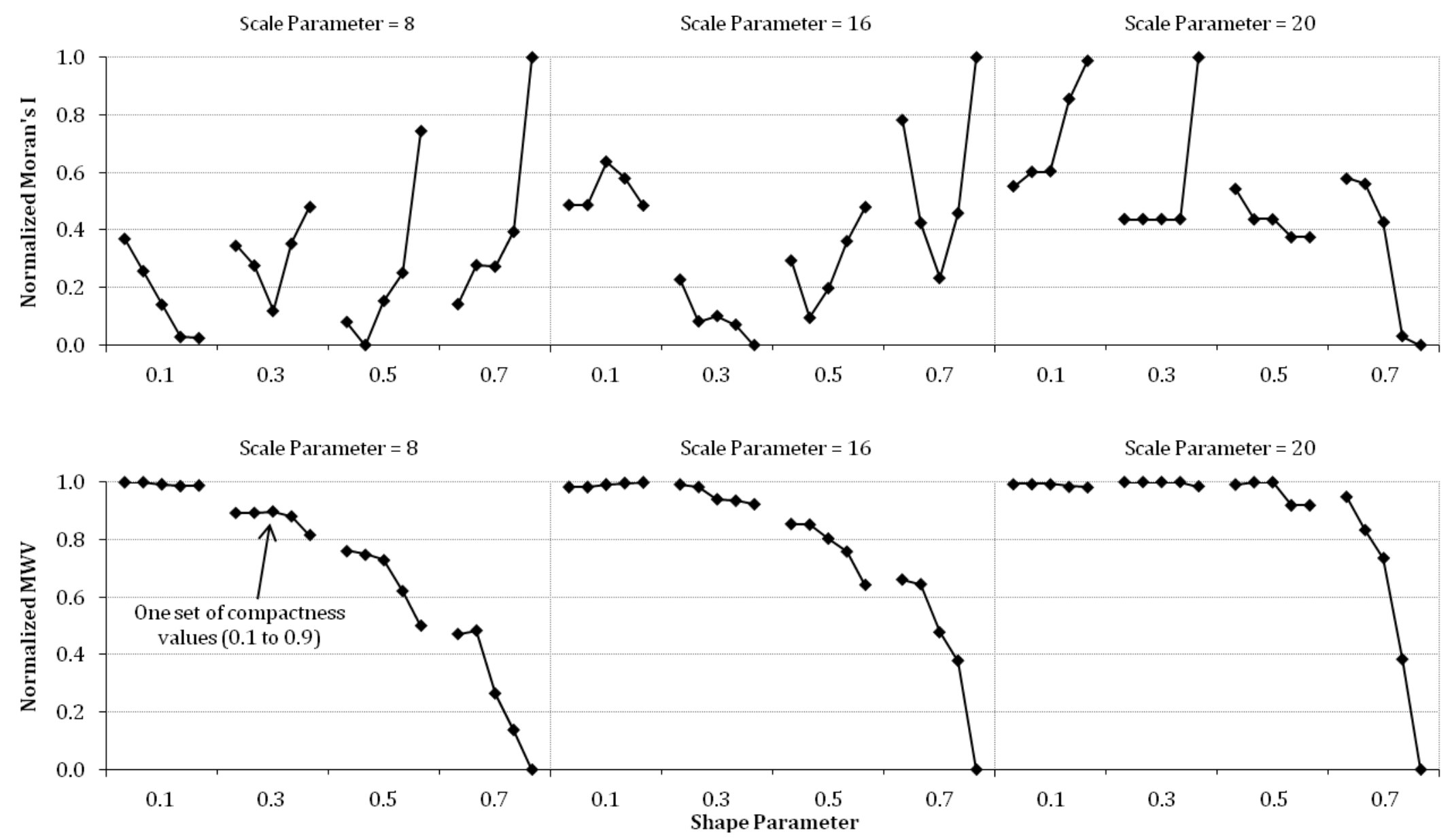

Figure 9. Normalized Moran's I index (top) and mean weighted variance (MVW, bottom) results from the image segmentation optimization analysis at three scale parameters. Each line segment represent compactness values 0.1 to 0.9 at a given shape parameter. 

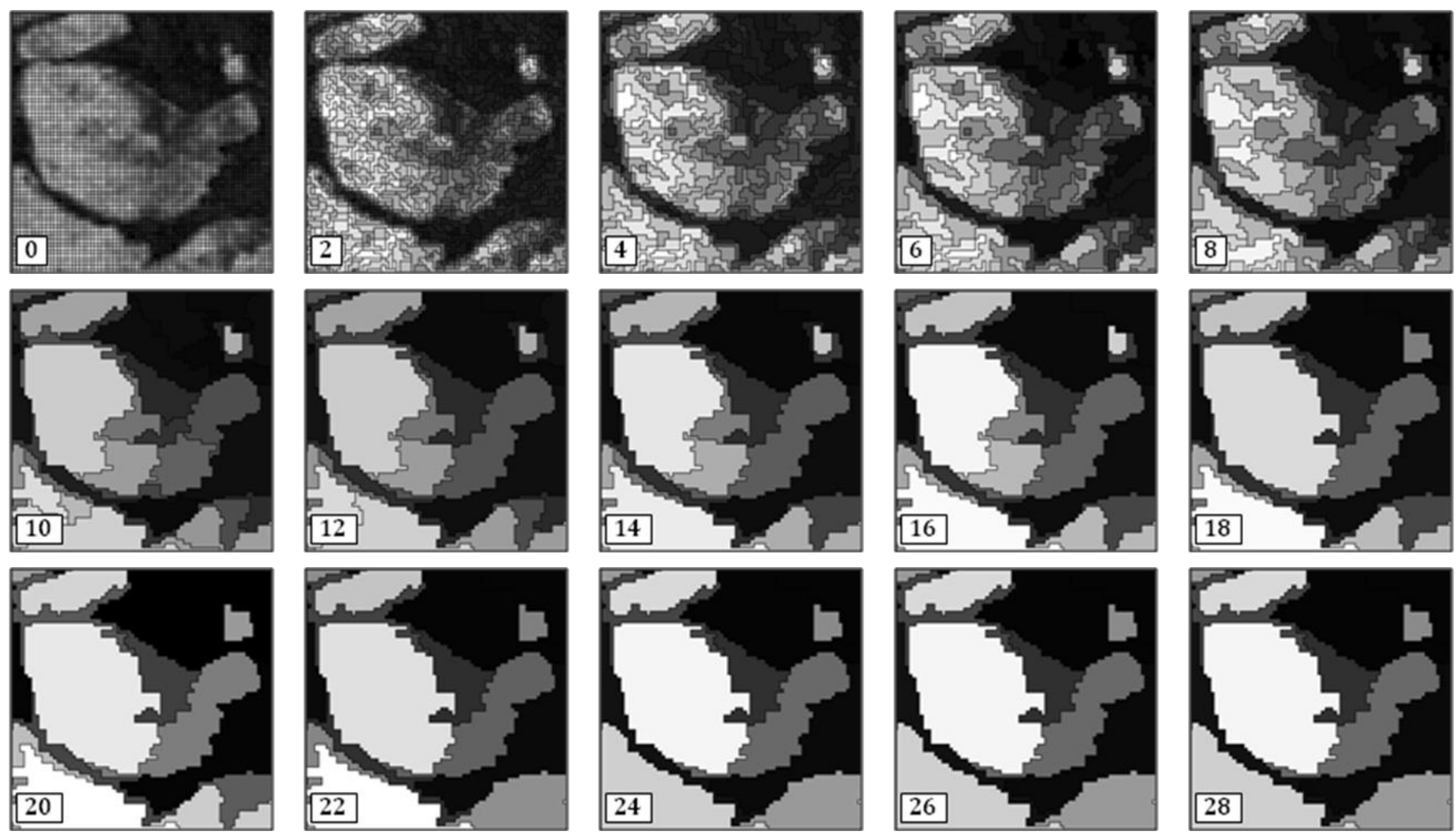

Figure 10. The final nested image hierarchy from a portion of the NDVIc image of Lava Cast Forest at incremental scale parameters $(0$, $2,4, \ldots, 28)$. The objects at each level are used to generate the next proceeding level. 
No combination of heterogeneity criteria simultaneously produce the highest normalized spatial autocorrelation and within-object variance measurements. The highest object function values for segmentations used to generate the image hierarchy range between 1.363 and 1.978 (Table 1). Compactness and shape parameters equal to 0.1 generate image segmentations with peak objective function values at scale parameters $2,4,6$, and 8 . In the subsequent 10 higher hierarchy levels, the objective function identifies 7 different optimal parameter combinations (Figure 11). At higher scale parameters, alternative compactness and shape parameters frequently generate identical results (e.g., scale parameter 14). Although no change in the number or arrangement of image objects exists between levels 26 and 28, normalization of the 20 candidate segmentations at these scale parameters causes different combinations of compactness and shape parameters to produce the highest objective function values. 
Table 1. Summary statistics for image segmentations possessing the lowest combined normalized Moran's I spatial autocorrelation coefficients and mean weighted variance (MWV).

\begin{tabular}{ccccccccccc}
\hline $\begin{array}{c}\text { Scale } \\
\text { Parameter }\end{array}$ & $\begin{array}{c}\text { Shape } \\
\text { Parameter }\end{array}$ & $\begin{array}{c}\text { Comp. } \\
\text { Parameter }\end{array}$ & $\begin{array}{c}\text { No. of } \\
\text { Objects }\end{array}$ & $\begin{array}{c}\text { Moran's I } \\
\text { Index }\end{array}$ & p-Value & Z-Score & $\begin{array}{c}\text { Normalized } \\
\text { Moran's I }\end{array}$ & MWV & $\begin{array}{c}\text { Normalized } \\
\text { MWV }\end{array}$ & $\begin{array}{c}\text { Objective } \\
\text { Function }\end{array}$ \\
\hline 28 & 0.5 & 0.5 & 31 & -0.090 & 0.582 & -0.551 & 0.376 & 42.437 & 1.000 & 1.376 \\
26 & 0.7 & 0.1 & 31 & -0.090 & 0.582 & -0.551 & 0.684 & 42.437 & 0.988 & 1.672 \\
24 & 0.7 & 0.1 & 32 & -0.087 & 0.592 & -0.536 & 1.000 & 42.081 & 0.815 & 1.815 \\
22 & 0.7 & 0.3 & 35 & -0.018 & 0.909 & 0.115 & 1.000 & 38.240 & 0.838 & 1.838 \\
20 & 0.3 & 0.9 & 40 & 0.014 & 0.669 & 0.427 & 1.000 & 33.137 & 0.985 & 1.985 \\
18 & 0.7 & 0.1 & 43 & 0.074 & 0.281 & 1.078 & 1.000 & 32.655 & 0.734 & 1.734 \\
16 & 0.1 & 0.5 & 71 & 0.097 & 0.117 & 1.567 & 0.639 & 23.976 & 0.993 & 1.632 \\
14 & 0.3 & $0.1,0.3$ & 85 & 0.152 & 0.011 & 2.549 & 0.932 & 23.387 & 0.963 & 1.895 \\
12 & 0.1 & 0.1 & 98 & 0.222 & 0.000 & 3.912 & 0.840 & 22.534 & 0.980 & 1.820 \\
10 & 0.5 & 0.9 & 138 & 0.320 & 0.000 & 6.567 & 0.960 & 21.848 & 0.403 & 1.363 \\
8 & 0.1 & 0.1 & 342 & 0.490 & 0.000 & 15.550 & 0.370 & 9.433 & 1.000 & 1.370 \\
6 & 0.1 & 0.1 & 571 & 0.587 & 0.000 & 24.262 & 0.693 & 7.029 & 0.999 & 1.692 \\
4 & 0.1 & 0.1 & 1,240 & 0.685 & 0.000 & 42.459 & 0.981 & 4.246 & 0.997 & 1.978 \\
2 & 0.1 & 0.1 & 4,929 & 0.801 & 0.000 & 102.723 & 0.504 & 0.998 & 0.979 & 1.483 \\
0 (pixels) & - & - & 25,600 & 0.934 & 0.000 & 298.136 & - & 0.000 & - & - \\
\hline
\end{tabular}



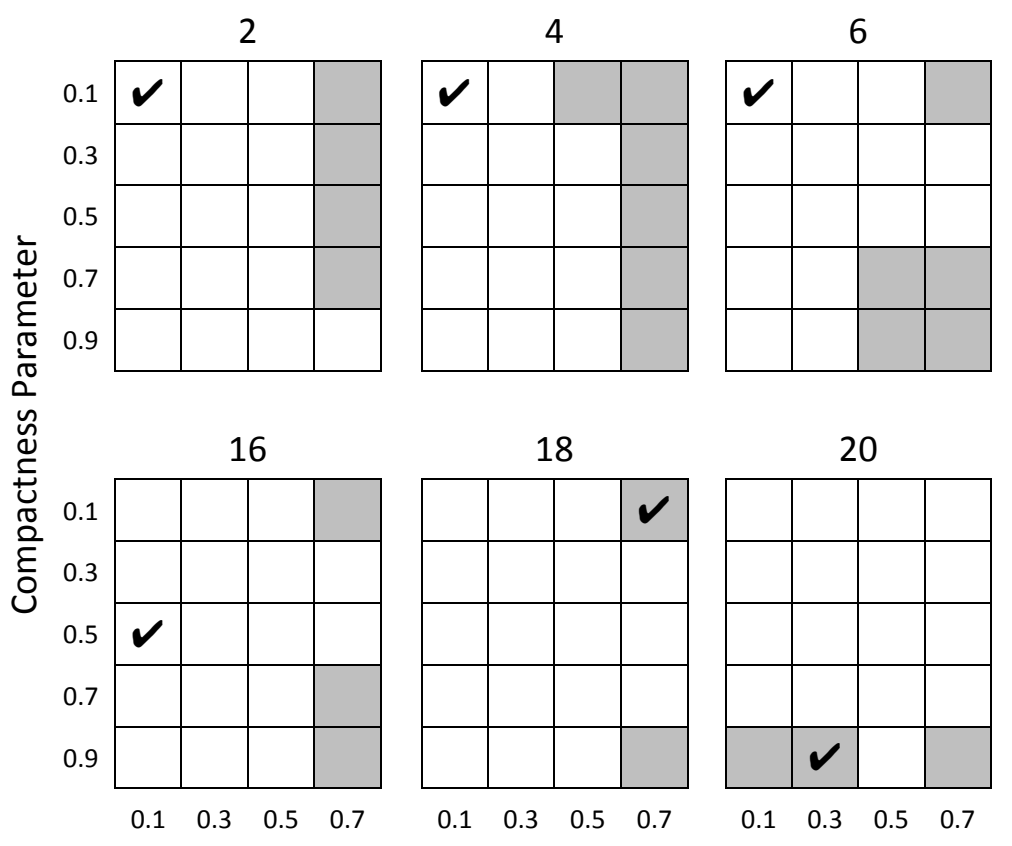

Scale Parameter
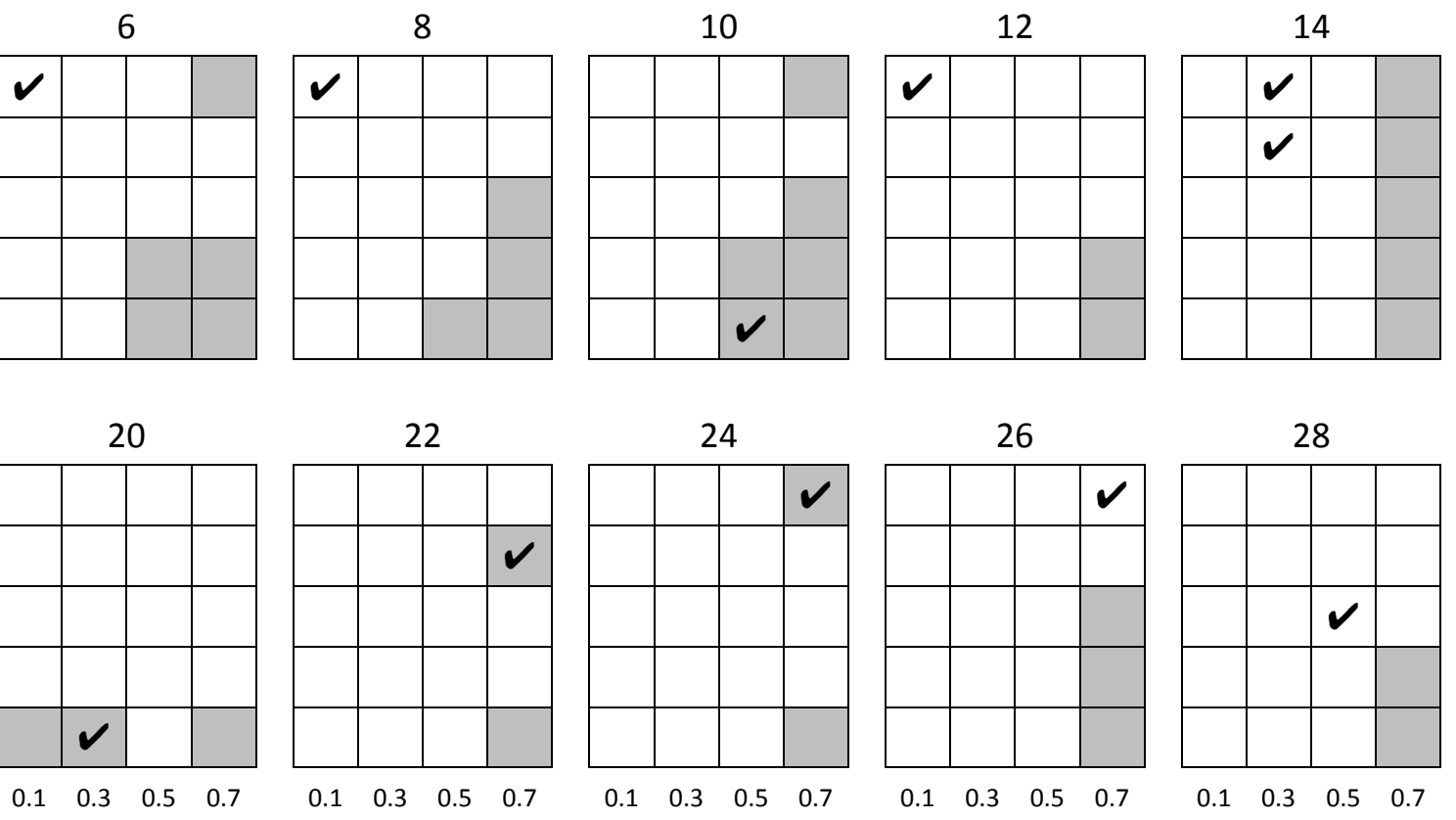

Shape Parameter

Figure 11. Image segmentation parameter combinations evaluated during construction of the NDVIc image hierarchy. Check marks indicate the optimal solution at each scale parameter. Grey boxes indicate a greater contribution to the objective function by Moran's I over the MWV. Image hierarchy development stopped when less than 30 image objects were created. 
Moran's I and MWV statistics for all combinations of shape and compactness parameters follow opposing gradients upward through the image hierarchy (Figure 12). The full range of Moran's I values decline steadily at each scale parameter increment, while those for MWV progressively increase. Segmentations used to generate hierarchy levels between scale parameters 0 and 14 have Moran's I estimates suggesting their constituent objects are significantly spatially autocorrelated $(\mathrm{p}<0.05)$. The Moran's I null hypothesis stating no significant clustering of values is rejected for image object networks in all higher levels (Table 1). Normalized Moran's I contributes a greater proportion to objective function values over normalized MWV in $35.7 \%$ of the hierarchy levels and $15.7 \%$ of the 280 total parameter combination evaluated in this study (Figure 11). Rapid changes in the within-object variance take place at scale parameters 10, 18, and between 22 and 24 when normalized Moran's I exceeds normalized MWV in the objective function calculation. These transitions are less obvious in the trend in spatial autocorrelation, and are followed by a corresponding sudden decrease in the number of image objects in the next higher level (Figure 13). 

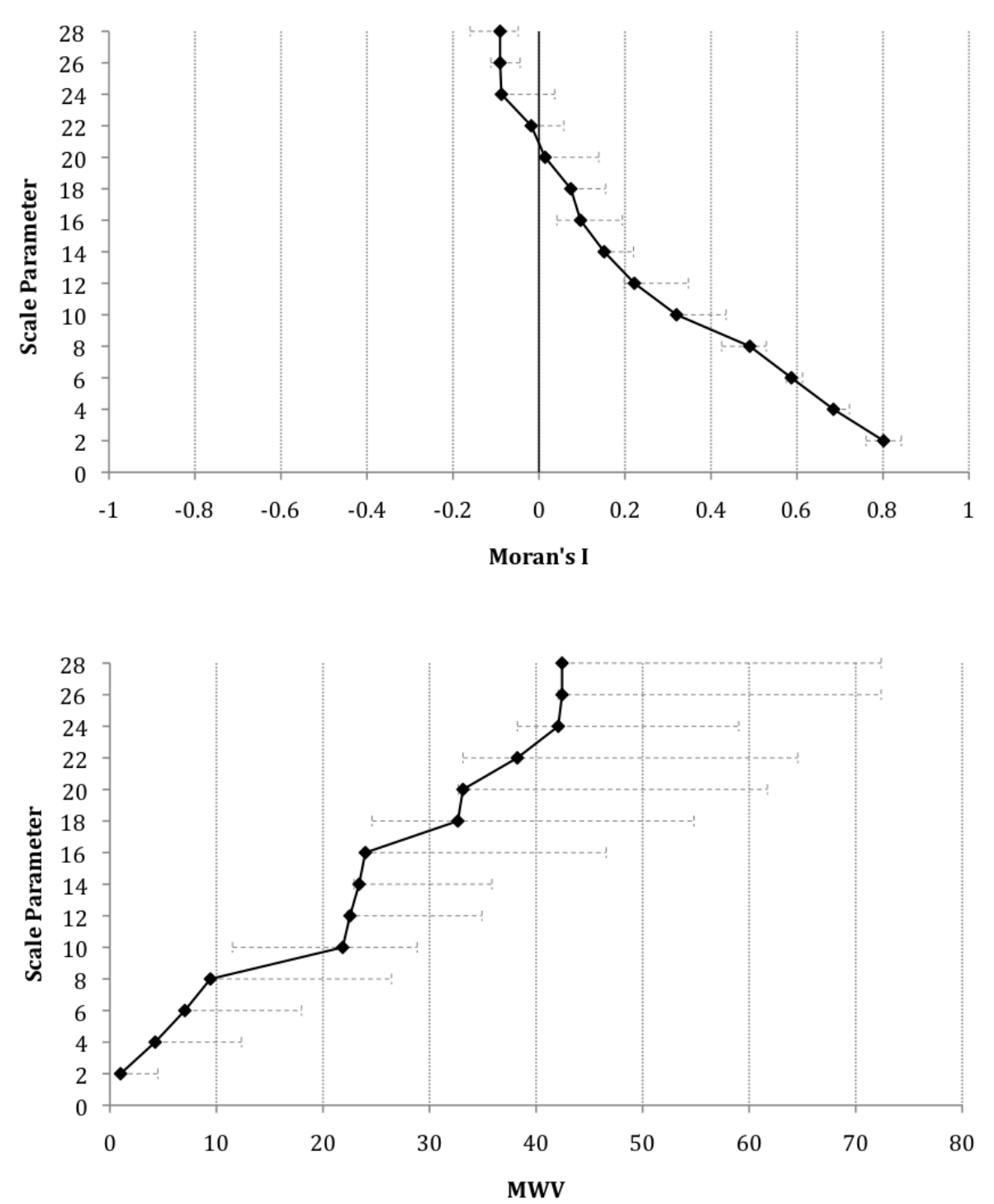

Figure 12. Trends in Moran's I (top) and MWV (bottom) for image object networks at each level in the image hierarchy. Horizontal min-max bars (dashed-lines) show the full range of values for the twenty segmentation parameter combinations evaluated at each scale parameter. 


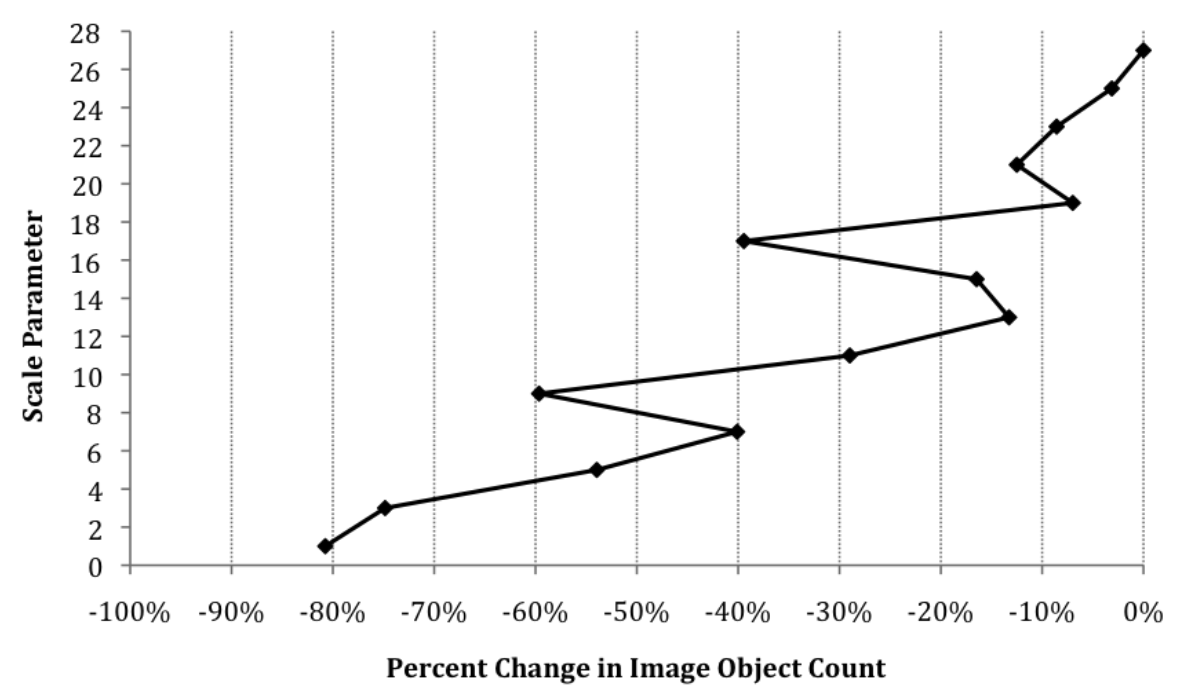

Figure 13. Percent change in the number of image objects between hierarchy levels.

\section{Distribution of Variance Across Scales}

Results of the geographical variance analysis identify four peaks of unusually high scale variance and the relative contribution to the percent sum of squares in the final rescaled NDVIc image hierarchy (Table 2). Presented graphically, the magnitudes of scale variance and percent sum of square values at corresponding scale parameters vary consistently relative to the hierarchy levels immediately above and below (Figure 14). The highest hierarchy level (scale parameter $=28)$ accounts for $78.9 \%$ of the total variability within the entire image hierarchy. The image segmentation at scale parameter 26 , which is reproduced exactly in the higher level, has a scale variance value equal to 0.0. Excluding the top level in the 
hierarchy, peaks in scale variance occur at scale parameters 8,16 , and 20 , which correspond to the levels immediately preceding those where rapid increases in MWV, Moran's I, and image object merging events are observed (Figures 12 and 13). Combined, these three levels constitute an additional $13.0 \%$ of the image hierarchy's total variability (Figure 15).

Table 2. Geographical variance analysis results for the NDVIc image hierarchy. Image objects at the lowest level in the hierarchy (scale parameter $=0$ ) are the individual pixels. The variability of the entire scene is expressed by the total sum of squares.

\begin{tabular}{cccccc}
\hline $\begin{array}{c}\text { Scale } \\
\text { Parameter }\end{array}$ & $\begin{array}{c}\text { Sum of } \\
\text { Squares }\end{array}$ & $\begin{array}{c}\text { Percent Sum of } \\
\text { Squares }\end{array}$ & $\begin{array}{c}\text { Degrees of } \\
\text { Freedom }\end{array}$ & $\begin{array}{c}\text { Expected Mean } \\
\text { Square }\end{array}$ & $\begin{array}{c}\text { Scale } \\
\text { Variance }\end{array}$ \\
\hline 28 & $4,055,298.2$ & 78.9 & 30 & $135,176.6$ & 153.2 \\
26 & 0.0 & 0.0 & 0 & 0.0 & 0.0 \\
24 & $9,109.7$ & 0.2 & 1 & $9,109.7$ & 0.6 \\
22 & $98,342.5$ & 1.9 & 3 & $32,780.8$ & 4.8 \\
20 & $130,635.6$ & 2.5 & 5 & $26,127.1$ & 14.5 \\
18 & $12,333.5$ & 0.2 & 3 & $4,111.2$ & 2.7 \\
16 & $222,190.6$ & 4.3 & 28 & $7,935.4$ & 32.4 \\
14 & $15,063.9$ & 0.3 & 14 & $1,076.0$ & 2.5 \\
12 & $21,835.4$ & 0.4 & 13 & $1,679.6$ & 5.3 \\
10 & $17,570.7$ & 0.3 & 40 & 439.3 & 3.5 \\
8 & $317,836.8$ & 6.2 & 204 & $1,558.0$ & 29.6 \\
6 & $61,538.9$ & 1.2 & 229 & 268.7 & 10.2 \\
4 & $71,238.5$ & 1.4 & 669 & 106.5 & 10.7 \\
2 & $83,148.5$ & 1.6 & 3,689 & 22.5 & 8.3 \\
0 & $25,549.1$ & 0.5 & 20,671 & 1.2 & 1.0 \\
\hline TOTAL & $5,141,691.8$ & 100.0 & 25,599 & - & -
\end{tabular}



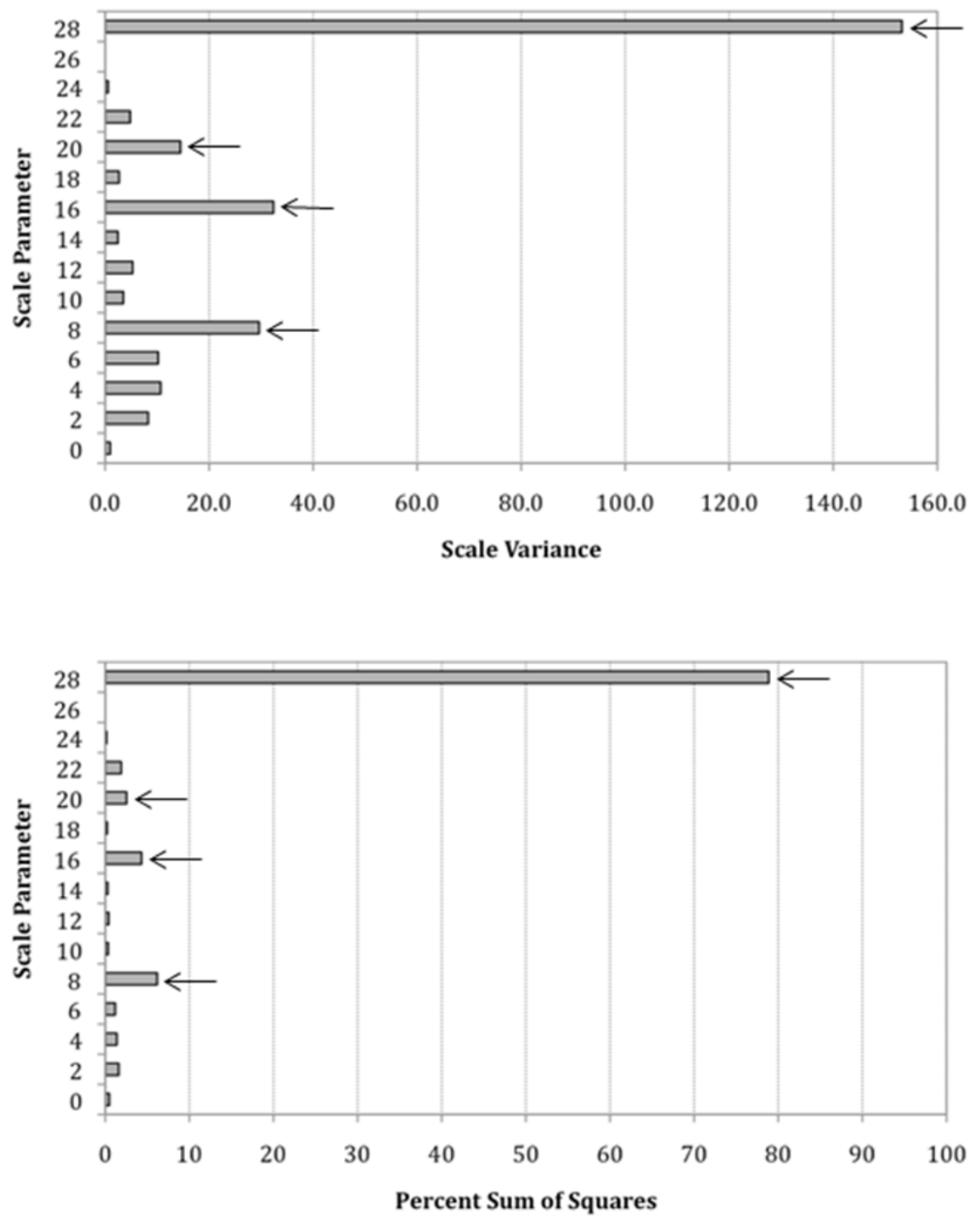

Figure 14. Scale variance (top) and percent sum of squares (bottom) for each level in the image hierarchy. 


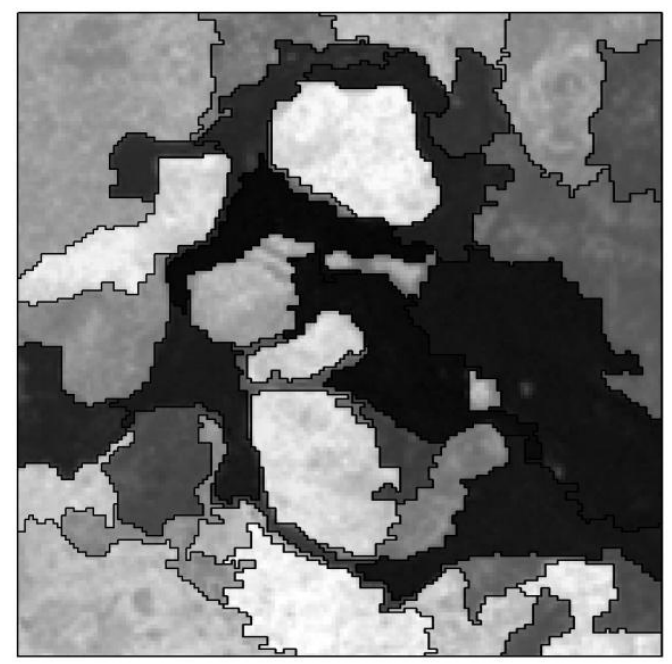

Scale Parameter $=20$
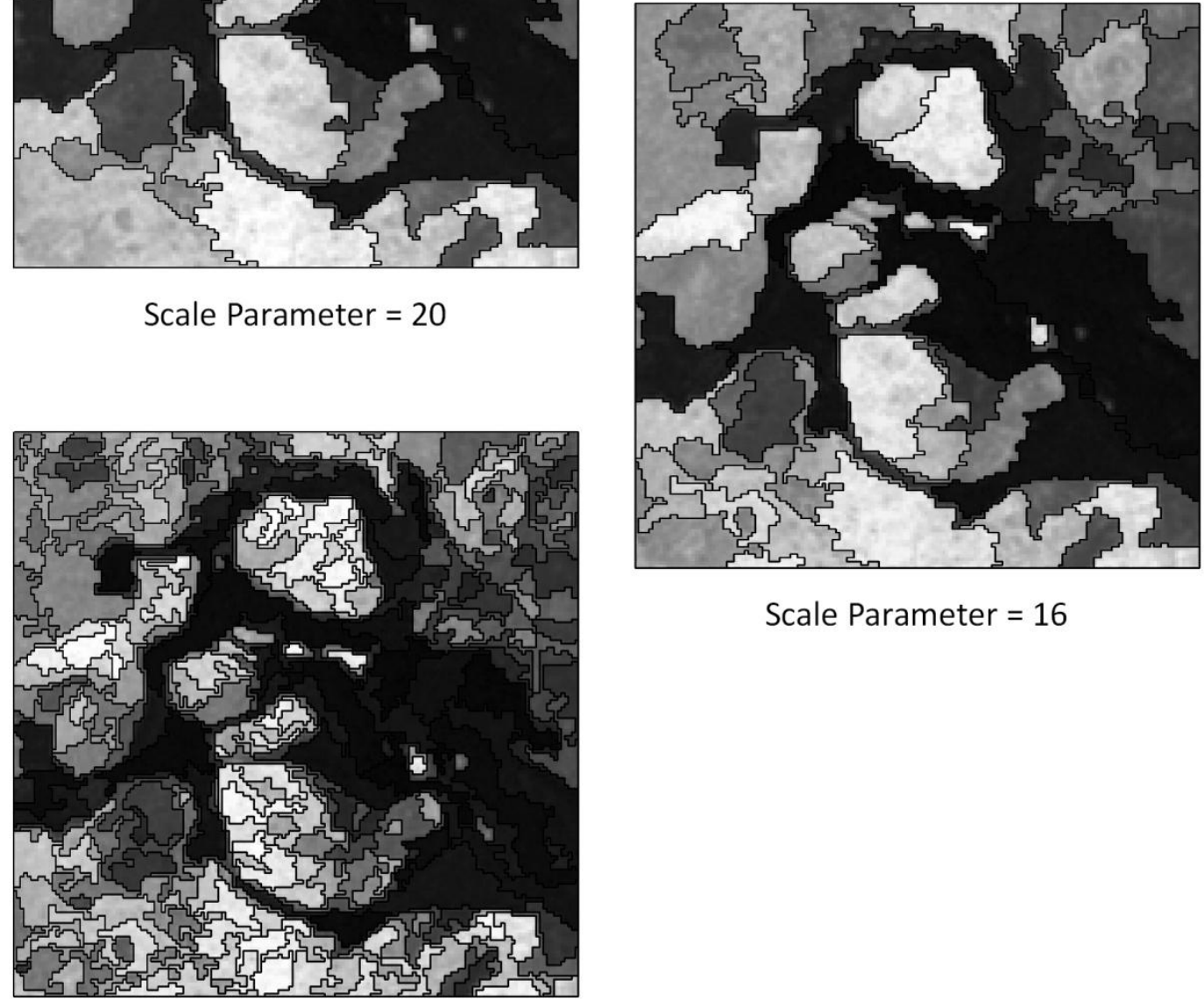

Scale Parameter $=16$

Scale Parameter $=8$

Figure 15. Landscape patterns at the three hierarchy levels identified by the image segmentation objective function and scale variance analyses. 


\section{DISCUSSION}

The complexity of heterogeneous landscapes is the result of numerous interacting biophysical processes that cannot be reduced into component parts without considering multi-scale linkages and the influence of higher-level constraints (Levin 1992, Wu and Loucks 1995). By considering individual patches in context with the entire landscape, the hierarchy patch dynamics framework addresses the challenge of characterizing and understanding the effects of patterns emergent in spatial heterogeneity across scales. The application of remote sensing and GIS to: 1) segment images at successively larger scales and 2) analyze resulting nested image hierarchies, is possible without preconceptions of patch structures or arrangements. This process is better informed by quantitative techniques that account for aggregation and scaling issues associated with the modifiable areal unit problem (MAUP).

\section{The Aggregation Problem}

The Definiens Developer 7 (DD7) heterogeneity criteria, compactness and shape, influence how individual pixels and objects are aggregated together at constant scale parameters. Recognizing that remotely sensed images of a particular landscape can vary considerably given differences in sensor specifications and 
environmental conditions at the time of acquisition, it is unreasonable to suggest that a single set of segmentation parameters are universally appropriate for all investigations. The objective function described in this research allows the effects of different parameter combinations to be systematically evaluated, provides a definitional rational for choosing between resulting image object mosaics, and ensures subsequent interpretations of landscape patterns are consistent between applications.

Interpretations of Moran's I and mean weighted variance (MWV) results at Lava Cast Forest (LCF) highlight the risks associated with subjectively selecting segmentation parameters. Although multiple combinations of parameters often produce identical results, this situation only once coincided with the maximum objective function in 14 scale parameters evaluated. By definition, the lowest DD7 heterogeneity criteria values would be expected to consistently produce objects with the lowest internal variance and greatest distinction between neighbors. Although this is the case initially, after reaching the fifth hierarchy level it becomes impossible to predict which subsequent combination of compactness and shape parameters will generate results best satisfying the objective function. High variability in the Moran's I component of the objective function between image object mosaics generated by alternative segmentation parameters is principally responsible for this uncertainty. Trends in MWV with incrementally larger compactness and shape values demonstrate that these parameters are influencing aggregations as intended by the DD7 segmentation protocol. Inconsistencies among 
segmentation parameter values across scales suggest resultant image objects likely represent real landscape entities and are not simply clustering the smallest progressions in spatial heterogeneity through scales.

Use of the Moran's I spatial autocorrelation coefficients allows aggregation effects to be more directly addressed (Marceau and Hay 1999). The efficacy of this statistic, however, becomes questionable as the number of image objects within a constant extent successively decreases at larger scales. Moran's I is a parametric statistic based on large-number theory requiring assumptions about the distribution and randomization of observations. In situations with small sample sizes, the likelihood of violating one of these assumptions increases, undermining the validity of results. The need for caution when interpreting Moran's I values is further warranted when substantially different spatial arrangements of landscape patches potentially possess equivalent spatial autocorrelation coefficients (Shortridge 2007).

In practice, image segmentations can be performed using incrementally larger scale parameters until pixels are contained within a single, landscape-scale object bounded by the study area extent. Concerns about the use of Moran's I component of the objective function, however, necessitated the creation of a stopping criterion. In this analysis, I chose to restrict the number of objects in the highest segmented hierarchy level when the following scale parameter produced less than 30 objects. As a consequence, the number of scale levels in the image hierarchy of LCF is limited to 14 segmented levels with greater than 30 objects. An 
alternative stopping criteria would be to convert Moran's I coefficients to Z-scores and evaluate whether candidate image object mosaics deviate significantly from a random pattern. This approach would have led to a cessation of hierarchical development of the rescaled NDVIc image after scale parameter 14 consisting of 85 image objects, and effectively reduced the range of scales investigated by one-half. Future scrutiny of the objective function described by Espindola et al. (2006) should consider the statistical provisions of the Moran's I spatial autocorrelation coefficient and explore how misinterpretations may result from potentially improper use of the statistic.

Aggregation issues related to the MAUP can lead to errors in the interpretation of individual landscape patches based on cumulative statistics for the entire mosaic. Developing nested image hierarchies requires all pixels, including those containing a mixture of land cover types, to be organized into discrete objects constrained by the boundaries of higher level objects and the analysis extent. One consequence of this rigid organizational framework is the delineation of landscape heterogeneity at or within transition zones between adjacent patches characterized by otherwise indeterminate boundaries. If the hierarchy theory framework is accepted as a reliable model for addressing the effects of scale on landscape organization, one possible explanation for edge objects is that they represent scaledependent transitions occurring at or within ecotonal gradients (Hay et al. 2001). Interpreting the structure and function of ecotones is difficult and of fundamental interest in landscape ecology (e.g., Gosz 1993). Monitoring the scales edge objects 
first appear, how long they persist, and when they coalesce with neighboring patches may offer insights into the interrelationships between multi-scale landscape patterns and ecosystem processes not possible from a single-scale perspective.

\section{The Scaling Problem}

Determining the scales that best approximate important landscape patterns is a critical first step in applying image segmentation and hierarchical image analysis for landscape characterization and ecological modeling. Quantitative methods for defining and detecting scale domains and thresholds are important for two reasons. First, they provide valuable insight into scaling issues. Second, they identify potentially important scales for evaluating multi-scale landscape patterns.

Hierarchy theory predicts scale thresholds can be recognized by peaks in the variance between aggregations of landscape features at specific scales, and are indicative of transitions in dominance from one set of pattern-process relationships to another (Wiens 1989, Wu 1999). Deviations in the progression of Moran's I and MWV within the rescaled NDVIc image hierarchy at LCF reveal possible locations of scale thresholds at scale parameters 8, 16, and 20 (Figure 12). Surges in the number of aggregation events (Figure 13) and peaks in scale variance identified with geographical variance analysis (Figure 14) also occur at these levels. Site characteristics at LCF described in several research projects (Hadley and Arabas in 
prep, Hrinkevich, 2005, Arabas et al. 2006, Karps 2006) combined with visual assessments of spatial patterns across scales supports the interpretation of these levels as the upper boundaries of scale domains. Beginning at the pixel level, neighboring image objects steadily cluster into larger objects, accumulating internal heterogeneity, until between-object differences are maximized at scale parameter 8 where the structure of the landscape pattern dramatically changes. Image objects below this threshold appear related to local differences in forest vegetation possibly explained by variations in disturbance frequency, succession rates, or other environmental factors (Arabas et al. 2006, Hadley and Arabas in prep). In addition, many objects in the lower domain occur at or within transition zones between larger objects. At scale parameter 10, the steady aggregation of objects resumes at a reduced rate with larger objects persisting through scales until changes in the dominant landscape pattern occur after scale parameters 16 and 20. Within these two domains, the majority of edge objects disappear and landscape patterns more closely coincide with topographic aspect and recently disturbed areas including the harvest units and blow-down (Figures 4 and 15). The rate of change in MWV after scale parameter 20 suggest the transition to the next higher domain may span two hierarchy levels (Figure 12).

One dominate landscape pattern not accounted for at any of the image hierarchy scale domains is the dichotomy between areas of relatively high forest vegetation cover, very low cover, and the lava flows (Figure 17). Two possible explanations may account for this oversight. The first involves the consequences of 
restricting the objective function analysis to segmentations producing more than 30 image objects. By preventing the hierarchy to develop incrementally up to the point of total amalgamation at the landscape-scale, only patterns generated by the range of scale parameters evaluated are observable and the study will fail to recognize important features or process-related controls at higher-scales (Wiens 1989). The high scale variance and relative contribution at scale parameter 28 provides evidence of a forest/lava scale domain (Figure 14).

Identification of a scale domain where landscape patterns adhere to the boundaries of the forest/lava interface may also be possible by expanding the study extent. Investigations conducted over larger analysis extents, however, inherently contain a greater proportion of spatial heterogeneity that can introduce unintended context from broader-scale landscape patterns and biophysical processes. For example, the high diversity in land-cover types within the region surrounding Newberry Crater could reduce the apparent significance of landscape patterns that characterize and influence the environment at LCF. 

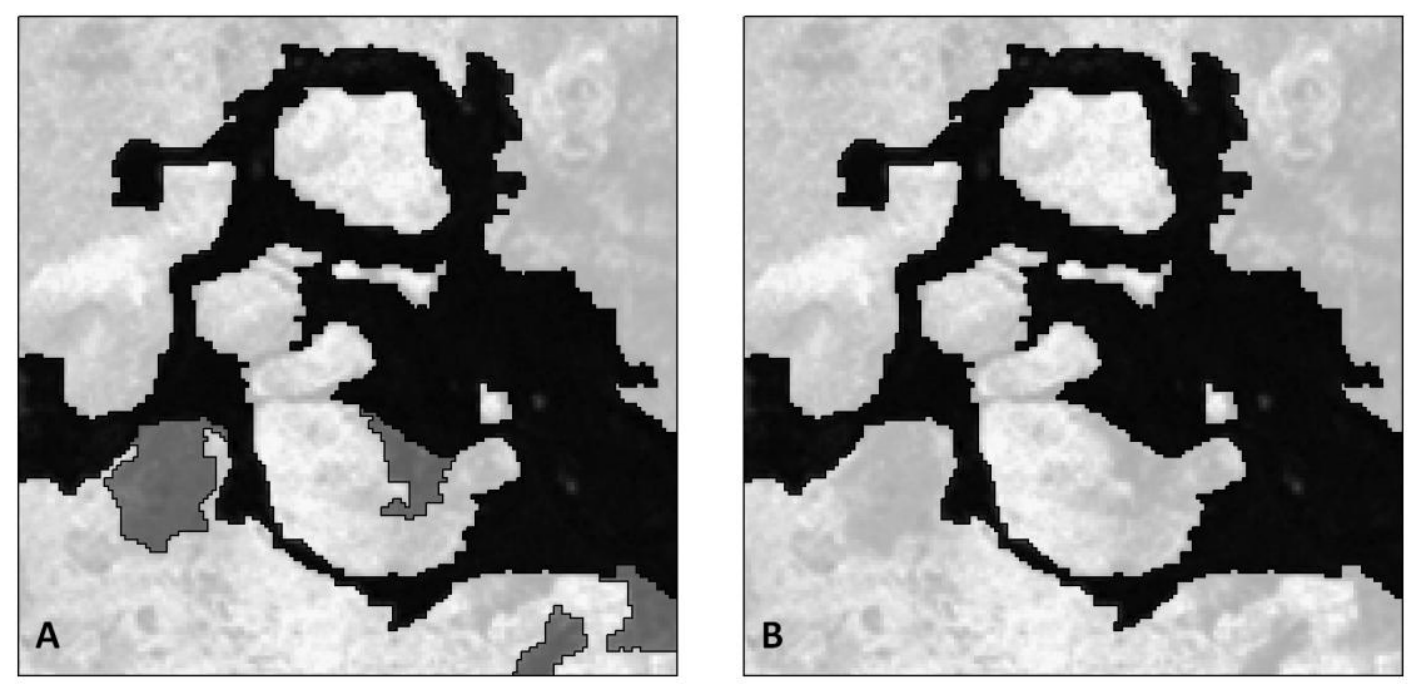

Figure 16. Two important landscape patterns unrealized in this analysis. Areas with lowest vegetation densities following disturbances from blow-down and timber harvesting are isolated in the image object mosaic shown in (A). The contrast between forest vegetation cover and the barren lava flows is depicted in (B).

Similar results derived from the objective function and geographical variance analyses suggest landscape heterogeneity in vegetation conditions at LCF can be generalized within three domains occurring between the analysis grain and extent (Figure 17). Some loss of information, however, would occur by removing the intervening levels. With the exception of scale parameter 26, whose objects are identical to those at scale parameter 28 , scale variance does not drop to zero between the threshold events. This observation implies the configuration of image objects and landscape heterogeneity vary continuously across scales. Future research providing a full accounting of the variance contributed by levels occurring 
between scale thresholds could justify their exclusion or expose important, landscape patterns previously unfamiliar in ecology (Hay et al. 2001).

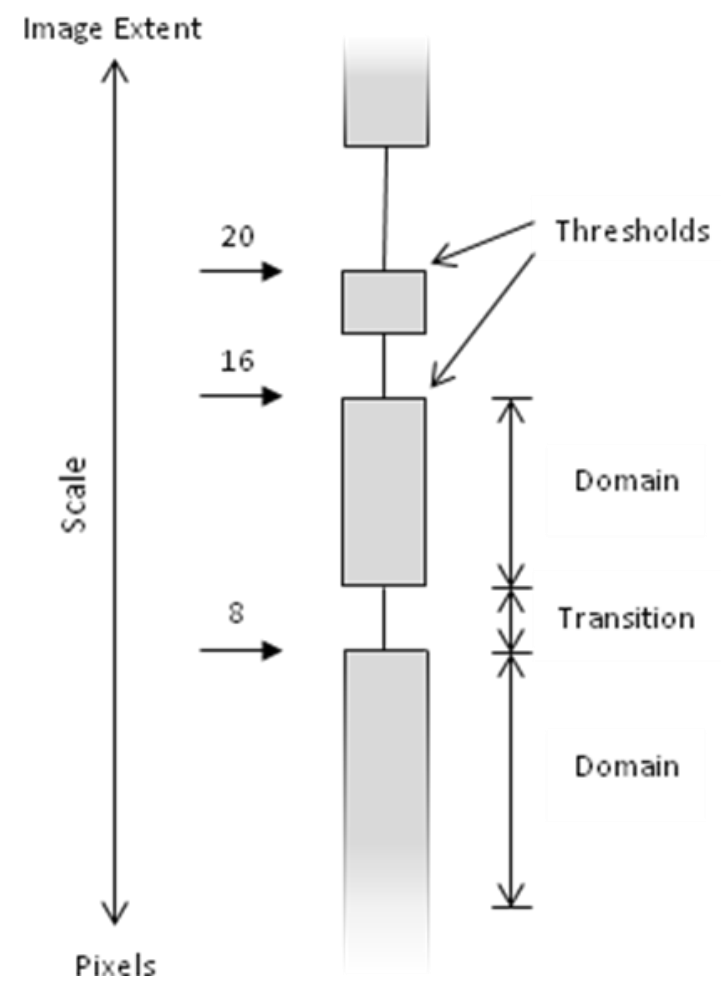

Figure 17. A restructuring of Wiens' (1989) figure illustrating the distribution of scale domains, transitions, and thresholds observed in the rescaled NDVIc image of LCF. The upper bounds of three scale domains were apparent from the geographical variance analysis at hierarchy levels 8,16 , and 20 . At these scales, spatial autocorrelation between neighboring objects exceeds mean within-object variance. Observation of scale domains at higher and lower scales are prevented by the chosen extent and image grain. 


\section{Methodological Considerations}

Analytical techniques designed to evaluate image segmentation parameters and facilitate investigations of multi-scale landscape patterns with remotely sensed imagery are most effective when they:

1. are objective in their approach,

2. account for all user-defined criteria,

3. appraise parameter settings in small increments, and

4. are sufficiently automated

Although the techniques presented in this paper generally satisfy these requirements, some technical issues still remain. The effectiveness of the Moran's I spatial autocorrelation coefficient, for example, remains a limiting factor when generating image segmentations with low numbers of objects. The full potential and possible idiosyncrasies of the geographical variance analysis method also deserve future scrutiny (Wu and Li 2009).

Each of the user-defined DD7 image segmentation criteria are adequately assessed between the objective function and geographical variance analyses with the exception that testing is restricted to a single image band. DD7 is capable of simultaneously segmenting large numbers of multi-resolution images, with each resultant image object possessing statistical and contextual attributes derived from all input bands. The expectation that a single image is suitable for studying the effects of scale in ecological systems is unwarranted given that no single biophysical 
variable can fully explain landscape complexity. Focus should begin with the objective function since it provides many of the same insights obtained with the geographical variance analysis, and is likely more amendable to multivariate datasets.

The choice to evaluate approximately half the range of image segmentation heterogeneity criteria values stemmed from an inability to completely automate the objective function workflow inside of DD7. Specifically, Moran's I calculations had to be performed outside of the DD7 environment causing interruptions in the process at each scale parameter increment. The possibility that a combination of compactness and shape parameters not addressed in this research might have produced the highest objective function value at a specific scale parameter evaluated is likely considering the high degree of variability observed in normalized Moran's I results. Testing scale parameters in single integer increments would have increased the number of hierarchy levels and more clearly resolved the distribution of variance across scales. 


\section{CONCLUSIONS}

One of the foundational goals of landscape ecology is to better understand how spatial heterogeneity is organized and its influence on biophysical processes and organism success (Levin 1992, Schlossberg and King 2009). Past research demonstrates that effective modeling, monitoring, and management of ecological systems and natural resources directly depend on the scales at which observations are made and interpreted (Whittaker et al. 2001, Dorner et al. 2002, Hall et al. 2004). In this paper, I addressed two fundamental challenges regarding the use of image segmentation and hierarchical image analysis in the evaluation of multi-scale spatial patterns in complex landscapes. These challenges are direct manifestations of the MAUP aggregation and scaling issues, and require conscientious attention in order to ensure results are repeatable, objective, and informative.

The first issue involves how interpretations of landscape patterns vary depending on how geospatial information is aggregated at a given scale. The objective function proposed by Espindola et al. (2006) provides a robust, nonarbitrary technique for operationally selecting high quality image objects by defining requirements based on the inherent variance and spatial autocorrelation properties of remotely sensed imagery. The inability to predict the outcome of segmentation results in this research using the object function emphasizes the importance of incrementally testing image segmentation parameter values. 
The second challenge considers the problem of selecting scales for addressing specific research questions. Adopting the hierarchy theory framework and conceptualizing the complex landscape at LCF across multiple scales facilitated the identification of three scale thresholds indicative of changes in dominant spatial patterns using geographical variance analysis (Moellering and Tobler 1972). The existence of potentially important, higher-level landscape patterns is suggested by the disproportional amount of scale variance contributed by the highest segmented level. Positive identification of these broad-scale patterns may be possible by reassessing the utility of the Moran's I coefficient for evaluating image segmentations producing low numbers of objects or adjusting the spatial analysis extent.

A limitation of this research is its characterization of multi-scale landscape patterns over a small geographic area using a single estimate of vegetation conditions derived from satellite imagery. Future studies should include an evaluation of the objective function and geographical variance analysis methods over larger extents and diverse landscapes with high-resolution multispectral imagery. Additional research is also necessary to determine whether each technique can be adjusted to include multivariate datasets and be more efficiently automated. These analyses should strive to incorporate adequate field-based information to help verify associations between image objects over numerous scales and real-world landscape features. The inclusion of ancillary geospatial data could be scaled with remote sensing estimates of environmental variables such as 
insolation, slope configuration, and disturbance history, to determine their correspondence with critical landscape pattern thresholds and scale domains.

Lastly, hierarchical image analysis can aid in the identification of appropriate scales for the development and application of ecological models. 


\section{LITERATURE CITED}

Arabas, K. B., Hadley, K. S., and Larson, E. R. 2006. Fire history of a naturally fragmented landscape in central Oregon. Canadian Journal of Forest Research 36:1108-1120.

Bellehumeur, C. and Legendre, P. 1998. Multiscale sources of variation in ecological variables: Modeling spatial dispersion, elaborating sampling designs. Landscape Ecology 13:15-25.

Benz, U. C., Hofmann, P., Willhauck, G., Lingenfelder, I., and Heynen, M. 2004. Multi-resolution, object-oriented fuzzy analysis of remote sensing data for GIS-ready information. ISPRS Journal of Photogrammetry \& Remote Sensing 58:239-258.

Bogaert, J. 2003. Lack of agreement on fragmentation metrics blurs correspondence between fragmentation experiments and predicted effects. Conservation Ecology 7:r6 [online] URL: http://www.consecol.org/vol7/iss1/resp6

Burnett, C. and Blaschke, T. 2003. A multi-scale segmentation/object relationship modelling methodology for landscape analysis. Ecological Modelling 168:233-249.

Cao, C.Y. and Lam, N. 1997. Understanding the scale and resolution effects in remote sensing and GIS. In Scale in remote sensing and GIS. Edited by D.A. Quattrochi and M.F. Goodchild. CRC Press LCL, Boca Raton, Florida, USA pp.27-55.

Clinton, N., Holt, A., Scarborough, J., Yan, L., and Gong, P. 2010. Accuracy assessment measures for object-based image segmentation goodness. Photogrammetric Engineering and Remote Sensing 76:289-299.

Crawley, M. J. and Harral, J. E. 2001. Scale dependence in plant biodiversity. Science 291:864-868.

Definiens Imaging, 2007. Definiens Developer 7. Definiens Imaging GmbH, München, Germany. 
Denny, M. W., Helmuth, B., Leonard, G. H., Harley, C. D. G., Hunt, L. J. H., and Nelson, E. K. 2004. Quantifying scale in ecology: Lessons from a waveswept shore. Ecological Monographs 74:513-532.

Dorner, B., Lertzman, K., and Fall, J. 2002. Landscape pattern in topographically complex landscapes: issues and techniques for analysis. Landscape Ecology 17:729-743.

Esch, T., Thiel, M. Bock, M., Roth, A., and Dech, S. 2008. Improvement of image segmentation accuracy based on multiscale optimization procedure. IEEE Geoscience and Remote Sensing 5:463-467.

Espindola, G. M., G. Camara, I. A. Reis, L. S. Bins, and A. M. Monteiro. 2006. Parameter selection for region-growing image segmentation algorithms using spatial autocorrelation. International Journal of Remote Sensing 27:3035-3040.

ESRI, 2010. ArcGIS 10 Beta. Environmental Systems Research Institute, Inc. (ESRI), Redlands, CA, USA.

Flanders, D., Hall-Beyer, M., and Pereverzoff, J. 2003. Preliminary evaluation of eCognition object-based software for cut block delineation and feature extraction. Canadian Journal of Remote Sensing 29:441-452.

Franklin, J. F. and Dryness, C. T. 1988. Natural Vegetation of Oregon and Washington. Oregon State University Press, Corvallis, OR. 452pp.

Gamon, J. A., Field, C. B., Goulden, M. L., Griffin, K. L., Hartley, A. E., Joel, G., Penuelas, J., and Valentini, R. 1995. Relationships between NDVI, canopy structure, and photosynthesis in three California vegetation types. Ecological Applications 5:28-41.

Gosz, J. R. 1993. Ecotone hierarchies. Ecological Applications 3:369-376.

Gotway, C. A. and Young, L. J. 2002. Combing incompatible spatial data. Journal of the American Statistical Association 97:632-648.

Hadley, K. S. and Arabas, K. B. (in prep). Forest structure, disturbance, and succession on forest isolates in central Oregon, USA.

Hall, O., Hay, G. J., Bouchard, A., and Marceau, D. J. 2004. Detecting dominant landscape objects through multiple scales: An integration of object-specific methods and watershed segmentation. Landscape Ecology 19:59-76. 
Hay, G. J., Marceau, D. J., Dubé, P., and Bouchard, A. 2001. A multiscale framework for landscape analysis: Object-specific analysis and upscaling. Landscape Ecology 16:471-490.

Hay, G. J., Dubé, P., Bouchard, A., and Marceau, D. J. 2002. A scale-space primer for exploring and quantifying complex landscapes. Ecological Modelling 153:27-49.

Hilker, T., Wulder, M. A., and Coops, N. C. 2008. Update of forest inventory data with lidar and high spatial resolution satellite imagery. Canadian Journal of Remote Sensing 34:5-12.

Hrinkevich, K.H., 2005. Forest edge dynamics in a naturally fragmented landscape in central Oregon, Masters Thesis, Portland State University, Portland, OR.

Iverson, L. R., Graham, R. L., and Cook, E. A. 1989. Applications of satellite remote sensing to forested ecosystems. Landscape Ecology 3:131-143.

Jelinski, D. E. and Wu, J. 1996. The modifiable areal unit problem and implications for landscape ecology. Landscape Ecology 11:129-140.

Jenny, H. 1994. Factors of Soil Formation. Dover Publications, Inc., New York. $281 \mathrm{pp}$.

Jensen, R. A. 2000. Roadside Guide to the Geology of Newberry Volcano, $3^{\text {rd }}$ edition. The Press Pro's, Bend, OR. 168pp.

Karps, J. M. 2006. Regeneration patterns and facilitation following blowdown in a self-replacing lodgepole pine (Pinus contorta) stand in central Oregon. Masters Thesis, Portland State University, Portland, OR.

Kotliar, N. B. and Wiens, J. A. 1990. Multiple scales of patchiness and path structure - a hierarchical framework for the study of heterogeneity. Oikos 59:253-260.

Lam, N. S-N. and Quattrochi, D. A. 1992. On the issues of scale, resolution, and fractal analysis in the mapping sciences. Professional Geographer 44:88-98.

Legrende, P. and Legrende L. 1988. Numerical Ecology, $2^{\text {nd }}$ Edition. Elsevier Press, Amsterdam. 853 p.

Levin, S. A. 1992. The problem of pattern and scale in ecology. Ecology 73:19431967. 
Marceau, D. J., Howarth, P. J., and Gratton, D. J. 1994. Remote sensing and the measurement of geographical entities in a forested environment. 1 . The scale and spatial aggregation problem. Remote Sensing of Environment 49:93-104.

Marceau, D. J. and Hay, G. J. 1999. Remote sensing contributions to the scale issue. Canadian Journal of Remote Sensing 25:357-366.

McBratney, A. B., Mendonca Santos, M. L., and Minasny, B. 2003. On digital soil mapping. Geoderma 117:3-52.

Moellering, H. and Tobler, W. 1972. Geographical variances. Geographical Analysis 4:34-64.

Möller, M., Lymburner, L., and Volk, M. 2007. The comparison index: A tool for assessing the accuracy of image segmentation. International Journal of Applied Earth Observation and Geoinformation 9:311-321.

Olden, J. D., Schooley, R. L., Monroe, J. B., and Poff, N. L. 2004. Context-dependent perceptual ranges and their relevance to animal movements in landscapes. Journal of Animal Ecology 73:1190-1194.

O’Neill, R. V., DeAngelis, D. L., Waide, J. B., and Allen, T. F. H. 1986. A Hierarchical Concept of Ecosystems. Princeton University Press, Princeton, New Jersey. $253 \mathrm{p}$.

Openshaw, S. 1977. A geographical solution to scale and aggregation problems in region-building, partitioning and spatial modelling. Transactions of the Institute of British Geographers 2:459-472.

Ord, J. K. and Getis, A. 1995. Local spatial autocorrelation statistics: Distributional issues and an application. Geographical Analysis 27:286-306.

Pal, N. R. and Pal, S. K. 1993. A review on image segmentation techniques. Pattern Recognition 26:1277-1294.

Peterson, R. G. and Groh, E. A. 1969. The ages of some Holocene volcanic eruptions in the Newberry Volcano area, Oregon. The Ore Bin 31:73-87.

Pickett, S. T. A. and Cadenasso, M. L. 1995. Landscape ecology: Spatial heterogeneity in ecological systems. Science 269:331-334. 
Pocewicz, A. L., Gessler, P., and Robinson, A. P. 2004. The relationship between effective plant area index and Landsat spectral response across elevation, solar insolation, and spatial scales in a northern Idaho forest. Canadian Journal of Forest Research 34:465-480.

Pohl, K. A., Hadley, K. S., and Arabas, K. B. 2006. Decoupling tree-ring signatures of climate variation, fire, and insect outbreaks in central Oregon. Tree-Ring Research 62:37-50.

Pribil, S. and Picman, J. 1997. The importance of using proper methodology and spatial scale in the study of habitat selection by birds. Canadian Journal of Zoology/Revue Canadien de Zoologie 75:1835-1844.

Riera, J. L., Magnuson, J. J., Vande Castle, J. R., and MacKenzie, M. D. 1998. Analysis of large-scale spatial heterogeneity in vegetation indices among North American landscapes. Ecosystems 1:268-282.

Rogerson, P. A. 2001. Statistical Methods for Geography. SAGE Publications Ltd, London. 236 p.

Schaefer, J. A. and Mayor, S. J. 2007. Geostatistics reveal the scale of habitat selection. Ecological Modelling 209:401-406.

Schlossberg, S. and King, D. I. 2009. Modeling animal habitats based on cover types: A critical review. Environmental Management 43:609-618.

Shortride, A. 2007. Practical limits of Moran's autocorrelation index for raster class maps. Computers, Environment and Urban Systems 31:362-371.

Stanton, S. and Arabas, K. B. 2009. Fuel and stand conditions in an isolated, unmanaged forest landscape in central Oregon. Annals of Forest Science 66:207.

Stoms, D. M. 1994. Scale dependence of species richness maps. Professional Geographer 46:346-358.

Todd, S. W., Hoffer, R. M., and Milchunas, D. G. 1998. Biomass estimation on grazed and ungrazed rangelands using spectral indices. International Journal of Remote Sensing 19:427-438.

Turner, D. P., Ollinger, S. V., and Kimball, J. S. 2004. Integrating remote sensing and ecosystem process models for landscape- to regional-scale analysis of the carbon cycle. BioScience 54:573-584. 
Turner, M. G. 1989. Landscape ecology: The effect of pattern on process. Annual Review of Ecology and Systematics 20:171-197.

Turner, M. G. 2005. Landscape ecology: What is the state of the science? Annual Review of Ecology, Evolution, and Systematics 36:319-344.

Ustin, S. L., Roberts, D. A., Gamon, J. A., Asner, G. P., and Green, R. 0. 2004. Using image spectroscopy to study ecosystem processes and patterns. BioScience 54:523-534.

Walsh, S. J., Moody, A., Allen, T. R., and Brown, D. G. 1997. Scale dependence of NDVI and its relationship to mountainous terrain. In Scale in remote sensing and GIS. Edited by D.A. Quattrochi and M.F. Goodchild. CRC Press LCL, Boca Raton, Florida, USA pp.27-55.

Ward, Jr., J. H. 1963. Hierarchical grouping to optimize an objective function. Journal of the American Statistical Association 58:236-244.

Wessman, C. A. 1992. Spatial scales and global change: Bridging the gap from plots to GCM grid cells. Annual Review of Ecology and Systematics 23:175200.

Whittaker, R. J., Willis, K. J., and Field, R. 2001. Scale and species richness: Towards a general, hierarchical theory of species diversity. Journal of Biogeography 28:453-470.

Wiens, J. A. 1989. Spatial scaling in ecology. Functional Ecology 3:385-397.

Woodcock, C. E. and Strahler, A. H. 1987. The factor of scale in remote sensing. Remote Sensing of Environment 21:311-332.

Wu, H. and Li, Z. H. 2009. Scale issues in remote sensing: A review on analysis, processing and modeling. Sensors 9:1768-1793.

Wu, J. and Loucks, O. L. 1995. From balance of nature to hierarchical patch dynamics: a paradigm shift in ecology. Quarterly Review of Biology 70:439466.

Wu, J. 1999. Hierarchy and scaling: Extrapolating information along a scaling ladder. Canadian Journal of Remote Sensing 25:367-380. 
Wu, J., Jelinski, D. E., Luck, M., and Tueller, P. T. 2000. Multiscale analysis of landscape heterogeneity: Scale variance and pattern metrics. Geographic Information Sciences 6:6-19.

Wu, J. and David, J. L. 2002. A spatially explicit hierarchical approach to modeling complex ecological systems: Theory and applications. Ecological Modelling 153:7-26.

Wu, J., Shen, W., Sun, W., and Tueller, P. T. 2002. Empirical patterns of the effects of changing scale on landscape metrics. Landscape Ecology 17:761-782.

Zhang, Y. J. 1996. A survey on evaluation methods for image segmentation. Pattern Recognition 29:1335-1346. 


\section{APPENDIX A \\ Example Geographical Variance Analysis}

This appendix provides an example of the calculations used to derive results for the geographical variance analysis described by Moellering and Tobler (1972). Real numbers are substituted for the mathematical notation used in the four-level example hierarchy shown in Figure 3 and the preceding text (Figure A1). Equation numbers match those on pages 18-21.
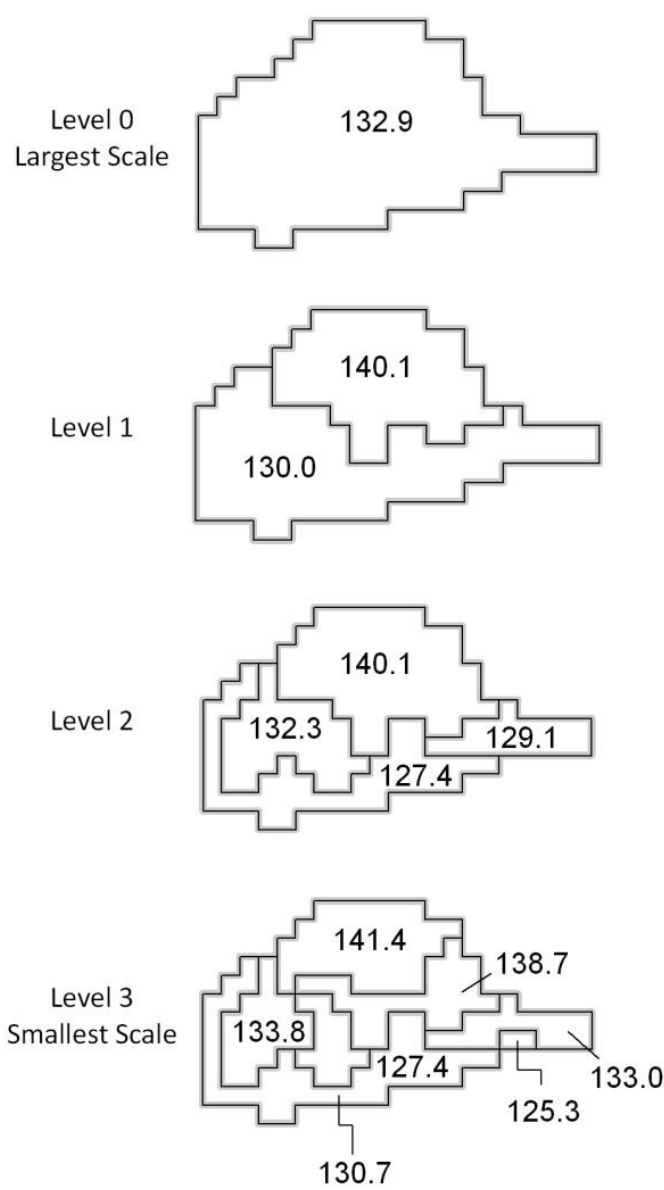

Figure A1. The example four-level hierarchy shown in Figure 3 with real numbers substituted for mathematical notation 
The $\mathrm{X}_{i j k}$ values and grand mean of the four-level example hierarchy in Figure

A is calculated by:

$$
\mu=\frac{133.8+130.7+127.4+133.0+125.3+141.4+138.7}{7}=132.9
$$

The squared deviations for the two Level 1 regions are:

$$
\begin{gathered}
\alpha_{1}=(130.0-132.9)^{2}=8.2 \\
\text { where } \overline{\mathrm{X}}_{1}=\frac{133.8+130.7+127.4+133.0+125.3}{5}=130.0 \\
\alpha_{2}=(140.1-132.9)^{2}=51.1 \\
\text { where } \quad \overline{\mathrm{X}}_{2}=\frac{141.4+138.7}{2}=140.1
\end{gathered}
$$

There are four aggregations in Level 2:

$$
\begin{gathered}
\beta_{11}=(132.3-130.0)^{2}=5.0 \\
\text { where } \overline{\mathrm{X}}_{11 .}=\frac{133.8+130.7}{2}=132.3 \\
\beta_{12}=(127.4-130.0)^{2}=6.9 \\
\text { where } \overline{\mathrm{X}}_{12 .}=\frac{127.4}{1}=127.4 \\
\beta_{13}=(129.1-130.0)^{2}=0.8 \\
\text { where } \overline{\mathrm{X}}_{13 .}=\frac{133.0+125.3}{2}=129.1 \\
\beta_{21}=(140.1-140.1)^{2}=0.0
\end{gathered}
$$




$$
\text { where } \quad \bar{X}_{21}=\frac{141.4+138.7}{2}=140.1
$$

The seven objects in Level 3 have the squared deviations:

$$
\begin{aligned}
& \gamma_{111}=(133.8-132.3)^{2}=2.4 \\
& \gamma_{112}=(130.7-132.3)^{2}=2.4 \\
& \gamma_{121}=(127.4-127.4)^{2}=0.0 \\
& \gamma_{131}=(133.0-129.1)^{2}=14.7 \\
& \gamma_{132}=(125.3-129.1)^{2}=14.7 \\
& \gamma_{211}=(141.4-140.1)^{2}=1.8 \\
& \gamma_{212}=(138.7-140.1)^{2}=1.8
\end{aligned}
$$

The total sum of squares for the hierarchy in Figure A is:

$$
\begin{aligned}
S S_{\text {Total }}=(133.8-132.9)^{2}+(130.7-132.9)^{2}+(127.4-132.9)^{2} \\
+(133.0-132.9)^{2}+(125.3-132.9)^{2}+(141.4-132.9)^{2} \\
+(138.7-132.9)^{2}=199.6
\end{aligned}
$$

The sum of squares across all subscripts for the three lower levels are:

$$
\begin{aligned}
& S S_{\alpha}=(5 \times 8.2)+(2 \times 51.1)=143.2 \\
& S S_{\beta}=(2 \times 5.0)+(1 \times 6.9)+(2 \times 0.8)+(2 \times 0.0)=18.6 \\
& S S_{\gamma}=2.4+2.4+0.0+14.7+14.7+1.8+1.8=37.8 \\
& \text { and } \quad S S_{\text {Total }}=143.2+18.6+37.8=199.6
\end{aligned}
$$

Right side of equal signs in (14) and (15) 
The percent sum of squares (PSS) for each level is then:

$$
\begin{gathered}
\operatorname{PSS}_{\alpha}=(143.2 / 199.6) \times 100=71.7 \% \\
\text { PSS }_{\beta}=(18.6 / 199.6) \times 100=9.3 \% \\
\text { PSS }_{\gamma}=(37.8 / 199.6) \times 100=19.0 \%
\end{gathered}
$$

The expected mean squares are:

$$
\begin{gathered}
E M S_{\alpha}=143.2 / 1=143.2 \\
E M S_{\beta}=18.6 / 2=9.3 \\
E M S_{y}=37.8 / 3=12.6
\end{gathered}
$$

Each level's scale variance component is:

$$
\begin{gathered}
S V_{\alpha}=143.2 / 1=143.2 \\
S V_{\beta}=18.6 / 2=9.3 \\
S V_{y}=37.8 / 3=12.6
\end{gathered}
$$

The majority of variance within the example hierarchy occurs at level 1 , followed by levels 3 and 2 (Figure A2). Level 1 accounts for nearly three-quarters of the total sum of squares. The extent, grain, and number of scales sampled are insufficient for determining whether the example hierarchy possesses a multi-scale structure. However, these results suggest the majority of information related to the structure of spatial patterns occurs at the lowest observed scale. 

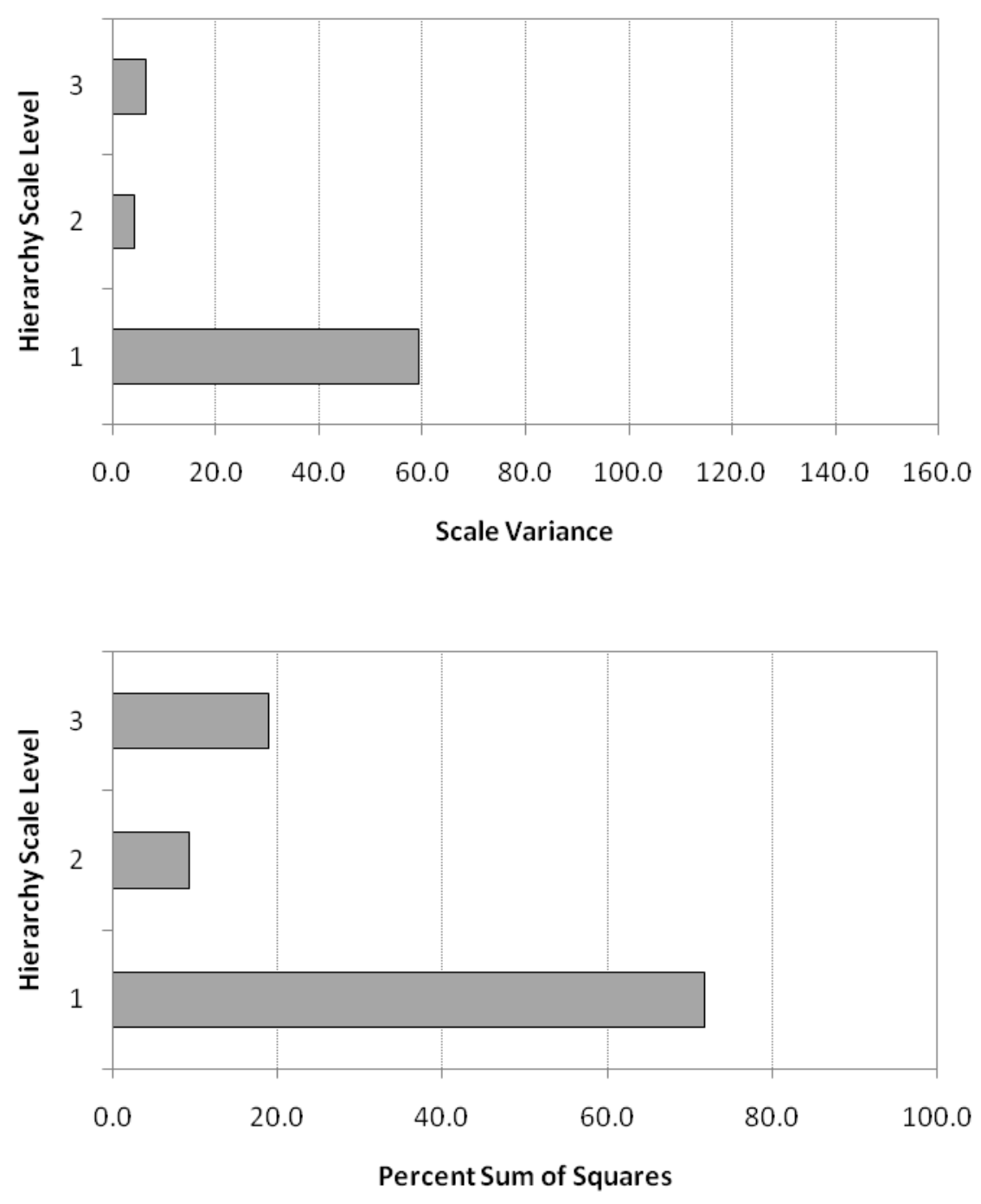

Figure A2. Scale variance (top) and percent sum of squares (bottom) for each level in the example image hierarchy. 